Sādhanā Vol. 38, Part 4, August 2013, pp. 591-620. (C) Indian Academy of Sciences

\title{
A comparative study of explicit and implicit modelling of subsegmental speaker-specific excitation source information
}

\author{
DEBADATTA PATI and S R MAHADEVA PRASANNA
}

Department of Electronics and Electrical Engineering, Indian Institute of Technology Guwahati, Guwahati 781 039, India

e-mail: debadatta@iitg.ernet.in; prasanna@iitg.ernet.in

MS received 3 September 2011; accepted 26 June 2013

\begin{abstract}
In this paper, the explicit and implicit modelling of the subsegmental excitation information are experimentally compared. For explicit modelling, the static and dynamic values of the standard Liljencrants-Fant (LF) parameters that model the glottal flow derivative (GFD) are used. A simplified approximation method is proposed to compute these LF parameters by locating the glottal closing and opening instants. The proposed approach significantly reduces the computation needed to implement the LF model. For implicit modelling, linear prediction (LP) residual samples considered in blocks of $5 \mathrm{~ms}$ with shift of $2.5 \mathrm{~ms}$ are used. Different speaker recognition studies are performed using NIST-99 and NIST-03 databases. In case of speaker identification, the implicit modelling provides significantly better performance compared to explicit modelling. Alternatively, the explicit modelling seem to be providing better performance in case of speaker verification. This indicates that explicit modelling seem to have relatively less intra and inter-speaker variability. The implicit modelling on the other hand, has more intra and inter-speaker variability. What is desirable is less intra and more inter-speaker variability. Therefore, for speaker verification task explicit modelling may be used and for speaker identification task implicit modelling may be used. Further, for both speaker identification and verification tasks the explicit modelling provides relatively more complimentary information to the state-of-the-art vocal tract features. The contribution of the explicit features is relatively more robust against noise. We suggest that the explicit approach can be used to model the subsegmental excitation information for speaker recognition.
\end{abstract}

Keywords. Speaker-specific excitation source information; subsegmental; explicit; implicit; LP residual; LF model.

\section{Introduction}

Speaker recognition involves finding out the speaker present in a given speech signal based on the information available in it (Campbell 1997). The speech signal is processed to extract some 
speaker-specific features for building the models and later use the same for testing. Speakerspecific characteristics in the speech signal is reflected due to the differences in, the dimensions and characteristics of the vocal tract and excitation source and learning habits of the speakers (Wolf 1972; Atal 1972). The vocal tract component represents the speaker information due to the unique resonant structure associated with each speaker and demonstrated to be containing significant speaker information (Furui 1981a). Vocal tract information is therefore being used for automatic speaker recognition (ASR) (Furui 2005). To date, vocal tract related cepstral features such as linear prediction cepstral coefficient $(L P C C)$ or mel frequency cepstral coefficient (MFCC) are the most dominant features for ASR (Furui 1981a; Davis \& Mermelstein 1980; Reynolds \& Rose 1995). These features mostly characterize the formant structure that depends on the shape and size of the vocal tract and hence provide good recognition performance (Furui 1981a; Davis \& Mermelstein 1980; Reynolds \& Rose 1995). If large amount of phonetically balanced speech data is available for speaker modelling, then LPCC or MFCC provides significant performance for ASR. Further, the performance of the cepstral features severely degrades under environmental variations that refers to the problem introduced by the noisy speech and variable recording channels (Zheng et al 2007; Reynolds 1994). Several compensation techniques have been applied to reduce the environmental effects. But, these techniques seem to be effective only in specified cases. Thus ASR systems based on vocal tract information seem to be suffering from the requirement of large data for modelling and poor performance due to varied environmental effects.

In applications where training and testing data are limited and of poor quality due to varied environmental effects, like mobile phone speech, there is a need for deriving robust features for speaker recognition task. Motivated by this the other component of the speech production system, that is, the excitation source has been explored. Both the physiological and behavioural aspects of the speaker like pith and intonation, respectively have been demonstrated to contain speaker information (Wolf 1972; Atal 1972). It was also shown that features derived from the excitation source require less amount of data for speaker recognition (Zheng et al 2007; Mary \& Yegnanarayana 2008; Prasanna et al 2006). Other attempts have also been made for exploring methods to model the speaker-specific information from the excitation source component (Prasanna et al 2006; Thevenaz \& Hugli 1995; Hayakawa et al 1997; Murty et al 2004; Yegnanarayana et al 2001; Murty \& Yegnanarayana 2006; Plumpe et al 1999; Wang et al 2009).

Vocal folds vibration depends on the size of the vocal folds (Deller et al 1993). For example, average rate of vocal folds vibration called as pitch is inversely proportional to the size of the vocal folds (Deller et al 1993). In general, the size of the vocal folds in male is larger than that of female and accordingly pitch of male (around 100-250 Hz) is lower than that of female (around 200-400 Hz) (Deller et al 1993). Since the physiological structure of the vocal folds is unique for a speaker, nature of vocal folds vibration may contain more speaker information apart from pitch. These include, nature of the periodicity of vibration, strength of the excitation at the instants of opening and closing and its variation from one instant to other. Unlike vocal tract features, it is difficult to represent all these information together in a single feature. The difficulty may be due to the dynamic nature of these information and may also be due to non-availability of the suitable signal processing tools.

Existing attempts on exploring the excitation signal mostly view the speaker-specific source information from three different levels called as subsegmental, segmental and suprasegmental levels (Prasanna et al 2006; Yegnanarayana et al 2001; Murty \& Yegnanarayana 2006; Pati \& Prasanna 2008, 2011, 2013). Subsegmental level mostly represents the source information present within one pitch period. This includes variation in the level of glottal flow within a glottal 
cycle and its timings like opening and closing instants. Segmental level mostly represents the source information present around two to three pitch periods. This includes periodicity of vocal folds vibration and its strength. Suprasegmental level represents the source information present over several pitch periods like pitch and excitation strength contours that reflects the learning habits of the speaker. These information can be modelled explicitly or implicitly for speaker recognition task. Explicit modelling refers to model the information by deriving the features from some parametric representation of the excitation signal. Since parameterization is involved, explicit modelling provides lossy but more compact representation of the source information (Pati \& Prasanna 2011, 2013). Implicit modelling refers to modelling the information using features derived from non-parametric representation of the excitation signal. For example, processing the blocks of linear prediction (LP) residual signal through auto associative neural network (AANN) or vector quantization (Prasanna et al 2006; Yegnanarayana et al 2001; Murty \& Yegnanarayana 2006; Pati \& Prasanna 2008). Implicit modelling is a lossless representation of the information but often computationally more intensive (Pati \& Prasanna 2011, 2013).

Existing attempts in exploring the source information for ASR may be treated under explicit or implicit approaches for modelling the subsegmental, segmental and suprasegmental levels information. The first attempt is the use of the pitch contours as the speaker information for ASR (Atal 1972). The pitch contour spans over several segments and hence may be viewed as explicit modelling of the suprasegmental level information. In Pati \& Prasanna (2011), both explicit and implicit approaches were applied to capture the suprasegmental level information of the source. In this study, for explicit modelling, pitch and excitation strength contours together were used. For implicit modelling, the LP residual is decimated by a factor 50 and processed in blocks of $250 \mathrm{~ms}$ with one sample shift. The purpose of decimation was to diminish other levels source information. It was shown that both modelling approaches of the suprasegmental level information provide almost equal recognition accuracy. In Huang et al (2008), Ezzaidi \& Rouat (2004) and Yegnanarayana et al (2005), pitch is used as an additional parameter along with vocal tract features like MFCC at frame levels, which seem to improve the performance. In this study, pitch is extracted for each segmental frame and appended to MFCC and hence may be viewed from explicit modelling of the segmental level source information. In Pati \& Prasanna (2013), both explicit and implicit modelling approaches are applied to capture the segmental level information and a comparative study is made. In this study, residual mel frequency cepstral coefficients $(R-M F C C)$ and mel power differences in subband spectra $(M-P D S S)$ features computed from subband source spectra were proposed for explicit modelling of the segmental level source information (Pati \& Prasanna 2010, 2013). For implicit modelling, the LP residual is decimated by a factor four and processed in blocks of $20 \mathrm{~ms}$. It was shown that with a small compromise in the recognition performance, explicit modelling approach provides more compact way of representing the segmental level information.

In Plumpe et al (1999), the subsegmental level information is modelled explicitly by estimating and modelling the glottal flow derivative (GFD) waveform from the speech signal and applied for ASR. The estimation of the GFD is decomposed into coarse and fine structures. The coarse structure represents the general flow shape and the fine structure represents the aspirations and other perturbations in the flow. GFD is modelled using the Liljencrants-Fant (LF) model to capture its coarse structure, while the fine structure of the flow derivative is represented through energy and perturbation measures. It was shown that both coarse and fine structures contain significant speaker information and helps $M F C C$ features to further improve the recognition performance. In Prasanna et al (2006) and Yegnanarayana et al (2001), the LP residual is processed in blocks of $5 \mathrm{~ms}$ with one sample shift to model the subsegmental level information. In Murty et al (2004), Murty \& Yegnanarayana (2006) and Pati \& Prasanna (2011) also, the analytic 
signal representation of the LP residual is processed in blocks of $5 \mathrm{~ms}$ with one sample shift to model the subsegmental level information. In these studies, the subsegmental level speaker information is implicitly captured using the AANN and Gaussian mixtures models (GMM) and demonstrated the presence of speaker information.

Speaker-specific information present at the subsegmental level of the excitation signal can be modelled by characterizing the glottal flow activities. The modulated air flow through the vocal folds due to their vibration is termed as glottal flow (Plumpe et al 1999; Ananthapadmanabha \& Yegnanarayana 1979). The area between the vocal folds is called as the glottis and hence the name glottal flow (Veldhuish 1998). Due to differences in the manner, speed and change in the rate of vocal folds vibration, the nature of the glottal flow varies from speaker to speaker. In some speakers, vocal folds never close completely (soft voice) and in other cases vocal folds close completely and rapidly (hard voice) (Plumpe et al 1999). Similarly, duration of opening and closing of vocal folds, the instants of glottal opening, and closing and the shape of the glottal flow also vary from speaker to speaker. Thus, the nature of the glottal flow contains speaker information and can be modelled by characterizing the glottal flow. Since, this information corresponds to one pitch period or glottal cycle, it may be viewed as subsegmental excitation information.

The way of characterizing the glottal flow is to measure the volume velocity of air flow through the glottis (Plumpe et al 1999). From the volume/pressure relationship, what we observe in the speech signal is the glottal air pressure that represents its derivative. Figure 1 shows the electro glotto gram (EGG) waveforms and their respective derivative of two male speakers, $M S-1$ and $M S$-2, collected from arctic database (Kominek \& Black 2004). The text of the speech signal remains same for fair comparison. EGG represents the actual glottal waveform that is produced by closing and opening of the vocal folds during the speech production. It can be observed that the EGG waveforms are different across speakers. The shape and duration of the waveforms are different across speakers. For example, the amplitude of the peaks in the glottal cycles in case of

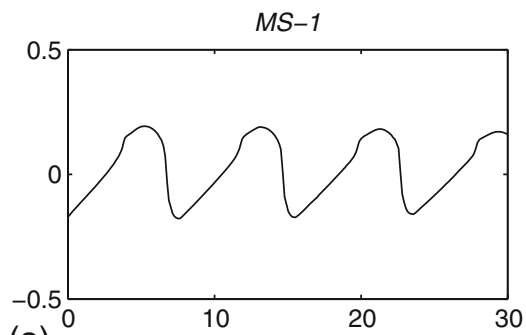

(a)
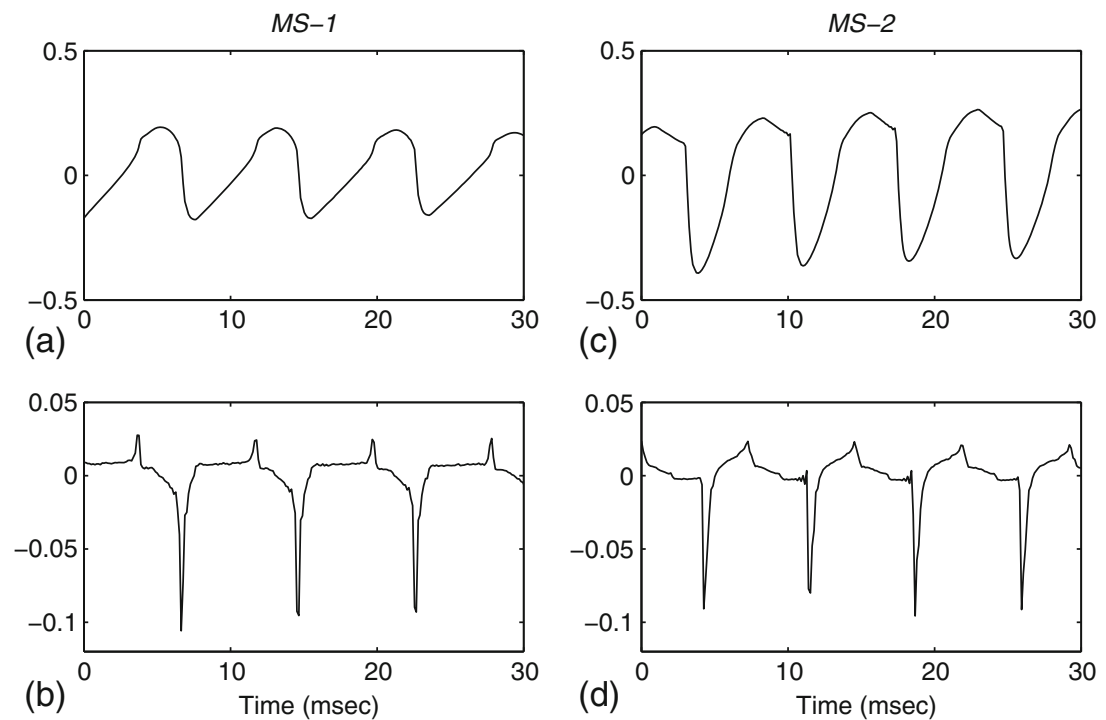

Figure 1. Examples of EGG and its derivative. (a), (c) EGG waveforms and their respective derivatives in (b) and (d) for speakers $M S-1$ and $M S$-2, respectively. 
speaker $M S$-2 is larger than $M S$-1. This shows that the amount of air flow in producing the speech for speaker $M S-2$ is relatively more. Further, the duration of the glottal cycles in case of speaker $M S-1$ is more than $M S$-2. As a result, the opening, closing and location of the peaks in each glottal cycle are different across speakers. This can also be clearly observed from the respective derivatives of the EGG waveforms. The first negative zero-crossing in each cycle of the EGG derivative represents the instant of maximum air flow and the location of the sharp peak represents the instants of rapid closing of the vocal folds. They are different across the speakers. Thus, GFD can also be used for explicit modelling of the subsegmental excitation information. For this, a method for accurate estimation of the GFD from the speech signal should be available. This is because, the EGG may not be available in many applications, since only speech is recorded.

As mentioned earlier, due to dynamic nature of the excitation, it is difficult to obtain a precise measurement of the GFD (Deller et al 1993). Therefore, analytical methods have been employed to model the GFD. Several methods have been proposed in the literature for analytic modelling of the GFD (Strik 1998). A comparative study on different methods for parameterization of the GFD has shown that, (in terms of smallest error) the LF model performs best (Strik 1998). The LF model has also been successfully used for speech synthesis and speaker recognition tasks (Plumpe et al 1999; Carlson \& Fant 1989). This shows that the LF model and its behaviour are well-known and hence may be useful for characterizing the GFD for explicit modelling of the subsegmental excitation information. In Plumpe et al (1999) and Karlsson (1988), it was shown that the parameterization of the GFD by LF parameters contain significant speaker information and also helps $M F C C$ features based speaker recognition system to further improve the performance. Thus, we prefer to use the LF model of the GFD cycle for modelling the subsegmental excitation information (Ananthapadmanabha \& Fant 1982). Unfortunately, the computational complexity involved in the LF parameters limits its use for modelling the glottal flow (Veldhuish 1998). It is expected that if a simplified algorithm is available to compute the LF parameters, then subsegmental excitation information can be modelled explicitly from the LF parameters with reduced computational complexity.

In this work, a simplified and approximate estimation method is proposed to compute the LF parameters from the LP residual and used them to model the subsegmental level information. In the proposed approach, the LF parameters are computed from blocks of LP residual samples of one glottal cycle duration. The blocks of LP residual samples are identified by locating the glottal closure and opening instants. Zero-frequency filtering approach is used to locate the glottal closure instants (GCI) (Yegnenarayana \& Murthy 2009). Glottal opening instants (GOI) are obtained from GCIs as the fixed fraction of the closed-phase intervals as proposed in Naylor et al (2007). First, the LF parameters computation is made with an assumption that the instants of the first zero crossing and the slope of the return phase are $50 \%$ and $10 \%$ of the GFD cycle interval, respectively. This assumption is made based on the concept that glottal closing is faster than its opening activities. The initial estimation of the LF parameters are optimized by using the constraint that the flow return to zero at the end of each glottal cycle. This constraint forces all parameters adjustment to be made concurrently (Qi \& Bi 1994). Then, the subsegmental level information is modelled explicitly by using the LF parameters and their dynamics. The effectiveness of the proposed approach is demonstrated by conducting different speaker recognition experiments. Finally, a comparison is made on implicit and proposed explicit modelling approaches of the subsegmental level information. For implicit modelling of the subsegmental level information, blocks of $5 \mathrm{~ms}$ with a shift of $2.5 \mathrm{~ms}$ LP residual samples are used (Pati \& Prasanna 2011). The comparison is made based on the computational complexity involved, recognition performance and their usefulness in providing the additional information to vocal tract information for ASR. 
The rest of the paper is organized as follows. Section 2 gives a brief review of the LF model of the GFD and then describes the proposed approach for computation of LF parameters from the LP residual for explicit modelling of the subsegmental level information. In this section, different speaker recognition studies are made using the features derived from the LF parameters to demonstrate the significance of the proposed approach. Section 3 describes the implicit modelling approach of the subsegmental excitation information and is used for speaker recognition studies. In section 4, a comparative study is made on explicit and implicit approaches for modelling subsegmental excitation information. In this section, the complimentary nature of the explicit and implicit features with the state-of-the-art vocal tract information in clean and noisy condition is also verified. The last section summarizes the present work with a mention on the scope for future work.

\section{Explicit modelling of subsegmental excitation information}

The glottal flow acts as the major excitation source to the vocal tract for the production of speech. In voiced speech, the glottal flow is periodic and one period of the glottal flow is called as the glottal pulse. As mentioned in the introduction section, the subsegmental excitation information is modelled explicitly from the GFD. The relation between the glottal flow and its derive for an ideal case is shown in figures $2 \mathrm{a}$ and $\mathrm{b}$, respectively. The glottal flow cycle is divided into three phases, called as, closed-phase, open-phase and return-phase. The interval during which the folds are closed and no flow occurs is called as the closed-phase. Practically, the vocal folds may not fully close and some air flow may always be present (Plumpe et al 1999). This may be due to very quick and incomplete closure of the vocal folds (Yegnanarayana \& Veldhuis 1998). However, in this phase the air flow is nearly constant and the folds are loosely coupled to the vocal tract. Due to differentiation, the constant air flow has negligible effect on the GFD and may be assumed to be zero (Veldhuish 1998). Thus, the closed-phase of the GFD may not provide any useful speaker-specific information.

The interval during which the vocal folds are open and there is an air flow through glottis is called as the open-phase. When the folds start to open, the interaction between the vocal folds and the vocal tract increases until a constant flow has been achieved. The variation in the

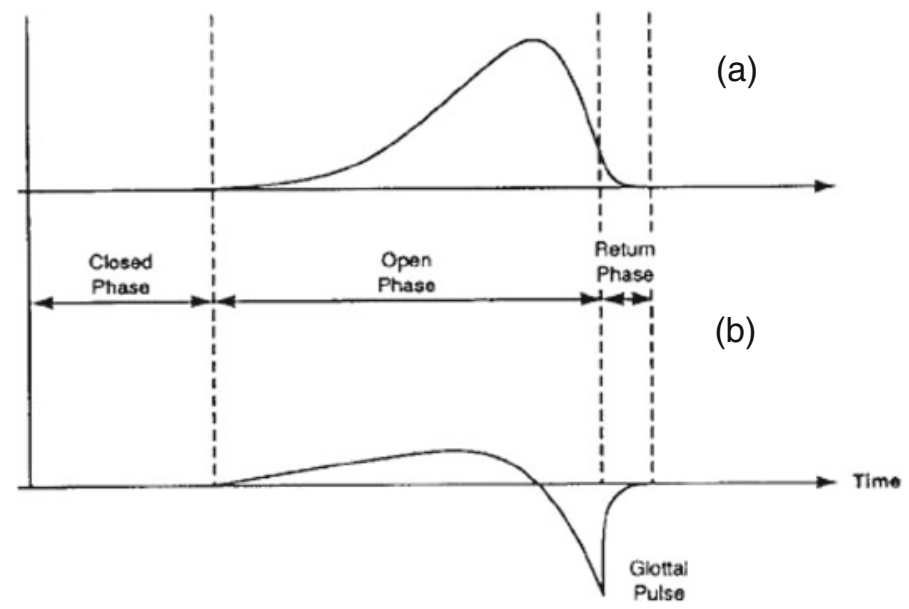

Figure 2. Relation between glottal flow and its derivative (Plumpe et al 1999). (a) Glottal flow. (b) GFD. 
shape of the glottal flow is therefore mostly due to the manner in which the glottis changes and the loading by the vocal tract (Plumpe et al 1999). Thus, variations in the GFD during the open-phase may contain significant speaker information. This may be attributed to the rate of increase of the flow, the maximum flow and the maximum rate of decrease of the flow and their corresponding instants. This can be easily observed from the GFD. For example, the shape of the GFD represents the rate of increase or decrease of the air flow, the zero-crossing represents the instants of the maximum air flow and its negative peak represents the maximum rate of decrease of the air flow. Since, the physical structure of the vocal folds differs from speaker to speaker, it is expected that the nature of the GFD cycle during open-phase may contribute to significant speaker information.

The interval from the instant of the maximum rate of decrease of the air flow to the instant of the zero air flow is called as the return-phase. This phase is particulary important, as it determines the amount of high frequency energy present in the glottal cycle (Plumpe et al 1999). The more rapidly the vocal folds close, the shorter the duration of the return-phase resulting in more high frequency energy. This can be observed from the exponential nature of the GFD during the return phase. Further, the air flow in the return-phase is generally considered to be of perceptual importance, because it determines the spectral slope (Veldhuish 1998). Thus, the nature of the glottal cycle during the return-phase is also expected to contribute significant speaker information.

In this section, we describe the method proposed to model the subsegmental level source information from LF model of the GFD. First, we give a brief introduction of the LF model and then describe the proposed approach for the estimation of its parameters. The usefulness of these parameters is demonstrated by speaker recognition studies.

\subsection{LF model}

The LF model of the glottal flow describes the GFD waveform in terms of exponentially growing sinusoid in the open-phase and a decaying exponential in the return-phase (Plumpe et al 1999; Deller et al 1993; Ananthapadmanabha \& Fant 1982). The shape of the model waveform is controlled by a set of analysis and synthesis parameters. The parameters derived from the inverse filtering of the speech signal are called as the analysis parameters. These include, the time of glottal opening and closing $\left(T_{o}, T_{e}\right)$, the maximum rate of the flow decrease and its location $\left(E_{e}, T_{e}\right)$. The parameters derived from the complex relationship of the GFD model are called as the synthesis parameters. These include, growth factor $(\alpha)$, flow decrease curvature and its nature $\left(w_{z}\right.$, $\beta$ ). The description of the seven LF parameters are given in table 1, (Plumpe et al 1999).

Table 1. Description of the seven parameters of the LF model of the GFD (Plumpe et al 1999; Veldhuish 1998; Qi \& Bi 1994).

\begin{tabular}{ll}
\hline$T_{o}$ & The time of glottal opening. \\
$T_{c}$ & Time of glottal closure. \\
$E_{e}$ & Absolute value of the maximum rate of glottal flow decrease. \\
$T_{e}$ & The time of maximum rate of glottal flow decrease. \\
$\alpha$ & The growth factor defined as the ratio of $E_{e}$ to maximum rate of glottal flow increase. \\
$w_{z}$ & Frequency that determines flow derivative curvature to the left of the first GFD zero \\
$\beta$ & $\quad$ crossing $\left(T_{z}\right), w_{z}=\frac{\pi}{T_{z}-T_{0}}$ (Plumpe et al 1999; Strik 1998). \\
$\beta$ & Exponential time constant that determines how quickly glottal flow \\
& derivative returns to zero after time $T_{e}$.
\end{tabular}


The mathematical expression for closed, open and return phases of one cycle of the GFD, $e_{L F}(t)$, from the synthetic LF model is given by the following equation (Plumpe et al 1999; Qi \& Bi 1994).

$$
\begin{array}{rlrl}
e_{L F}(t) & =0, & 0 & \leq t<T_{o} \\
& =E_{o} e^{\alpha\left(t-T_{o}\right)} \sin \left[w_{z}\left(t-T_{o}\right)\right], & T_{o} \leq t<T_{e} \\
& =-\frac{E_{e}}{\beta T_{a}}\left[e^{-\beta\left(t-T_{e}\right)}-e^{-\beta\left(T_{c}-T_{e}\right)}\right], & T_{e} \leq t<T_{c} .
\end{array}
$$

In Eqn. 1, $E_{o}$ is an arbitrary gain constant and $T_{a}$ (time constant of the return phase) is the the projection of the modelled GFD at $t=T_{e}$ in the time axis (Veldhuish 1998; Qi \& Bi 1994). In the LF model of the GFD, the closed phase is assumed to be zero. This will not affect in modelling the GFD. Because, as mentioned earlier, the small air flow during closed phase have less effect on the GFD. A synthetic glottal flow and its derivative modelled by the LF parameters are shown in figure $3 \mathrm{a}$ and $\mathrm{b}$, respectively. It can be observed from this figure that, $T_{o}, T_{e}, E_{e}$, $\alpha, w_{z}$ and $E_{o}$ characterize the open-phase and $E_{e}, \beta$, and $T_{c}$ characterize the return-phase. It should be noted here that the LF model also includes the parameter $T_{a}$ that determines spectral tilt which is perceptually important (Veldhuish 1998). All these parameters needs to be supplied explicitly for modelling the GFD. Some parameters like $T_{o}, T_{c}, T_{e}$ and $E_{e}$ can be obtained from the LP residual by locating the glottal opening and closing instants using recently proposed

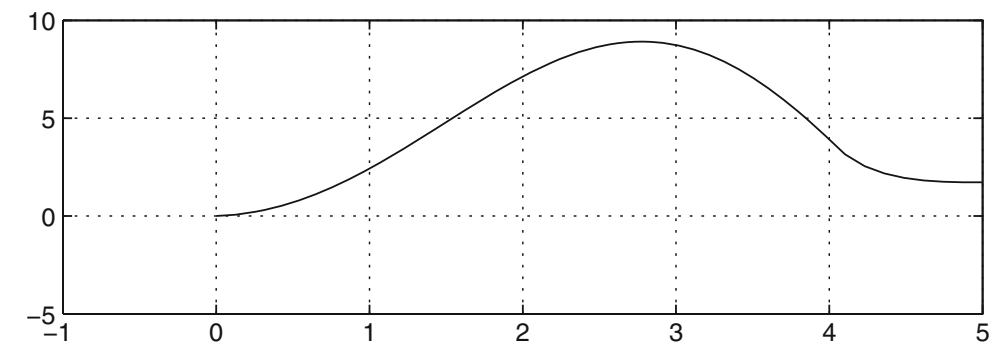

(a)

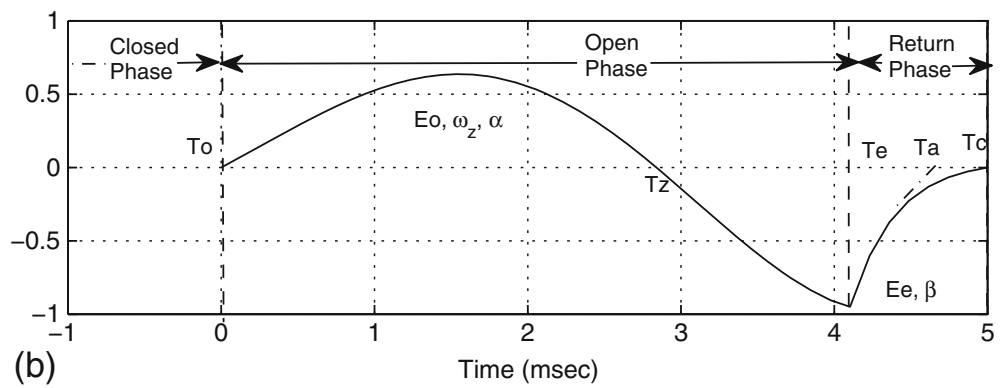

Figure 3. Typical glottal flow and GFD waveforms with the parameters of the LF model. (a) glottal flow. (b) GFD of the flow in (a). The GFD is modelled by Seven LF parameters. Three of the parameters $\left(E_{o}\right.$, $w_{z}$, and $\left.\alpha\right)$ describe the shape of the glottal flow during nonzero flow $\left(T_{o}-T_{e}\right)$. The two parameters $\left(E_{e}, \beta\right)$ describe the shape of the glottal flow during most negative glottal flow derivative and glottal closure $\left(T_{e}\right.$ $\left.T_{c}\right)$. The other two parameters $\left(T_{e}\right.$ and $\left.E_{e}\right)$ describe the time and amplitude of the most negative peak of the glottal flow derivative. 
zero-frequency filtering method (Pati \& Prasanna 2011; Yegnenarayana \& Murthy 2009). The computation of the other parameters are computationally tedious due to noise like nature of the LP residual and the complex relationship among the LF parameters. A simplified method for the approximate computation of these parameters from the LP residual is described below.

\subsection{Computation of LF parameters}

The LF parameters are computed for each glottal cycle individually. In section 2.1, we observe that amplitude of the GFD during the closed phase is zero and the parameters used to describe the LF model of the GFD are associated with the open and return phases. Thus, the effective GFD cycle can be assumed to be starting at the instant of the glottal opening and ends at the instant of the glottal closing. By considering every glottal cycle starts at $t=0$ and ends at $t=T_{c}$, the mathematical expression of the LF model for an individual GFD cycle given in Eq. (1) can be modified as,

$$
\begin{aligned}
e_{L F}(t) & =E_{o} e^{\alpha(t)} \sin \left[w_{z}(t)\right], & & \leq t<T_{e} \\
& =-\frac{E_{e}}{\beta T_{a}}\left[e^{-\beta\left(t-T_{e}\right)}-e^{-\beta\left(T_{c}-T_{e}\right)}\right], & & T_{e} \leq t<T_{c} .
\end{aligned}
$$

Further, at $t=T_{e}, e_{L F}(t)=-E_{e}$. Thus, we have from Eq. (2)

$$
\begin{aligned}
E_{o} e^{\alpha T_{e}} \sin \left(\omega_{z} T_{e}\right) & =-E_{e} \\
\Rightarrow \alpha & =\frac{1}{T_{e}} \ln \left[-\frac{E_{e}}{E_{o} \sin \left(\omega_{z} T_{e}\right)}\right]
\end{aligned}
$$

and from Eq. (3)

$$
\begin{aligned}
-\frac{E_{e}}{\beta T_{a}}\left[1-e^{-\beta\left(T_{c}-T_{e}\right)}\right] & =-E_{e} \\
\Rightarrow 1-e^{-\beta\left(T_{c}-T_{e}\right)} & =\beta T_{a} .
\end{aligned}
$$

Eq. (4) and (5) indicate that the relationship among the LF parameters have no close form solution and may be solved iteratively using nonlinear least squares algorithms like Gauss-Newton or Newton-Raphson algorithms (Plumpe et al 1999; Iseli \& Alwan 2000). But, these algorithms are computationally intensive and are not adequate when the minimum error is large (Plumpe et al 1999). To avoid this difficulty, adaptive nonlinear least squares regression techniques have been proposed (Plumpe et al 1999). The difficulty in this approach is that, the estimated parameters may be too close to their bound that leads to physically unrealistic conditions. For example, $E_{o}$ taking a negative value or a value near to zero. Thus, iterative approach of estimating the parameters from the actual LF model may not seem to be useful. To avoid the risk of unrealistic conditions and also to reduce the computational complexity, we prefer to estimate the parameters from a simplified LF approximation model. In Qi \& Bi (1994), one such model is proposed, where it is assumed that the return flow is relatively faster, for example, $\beta\left(T_{c}-T_{e}\right) \gg 1$. This assumption helps us in reducing the nonlinear Eq. (5) to a simplified form as given below.

$$
\begin{aligned}
\beta\left(T_{c}-T_{e}\right) & \gg 1 \\
\Rightarrow e^{-\beta\left(T_{c}-T_{e}\right)} & \simeq 0 .
\end{aligned}
$$

Thus, from Eq. (5) and Eq. (6),

$$
\beta T_{a}=1 .
$$


In the above assumption a constraint is imposed that the GFD returns to zero at the end of each cycle. Such that,

$$
\int_{0}^{t} e_{L F}(t) d t=0
$$

The above constraint forces all the parameters adjustment to be made concurrently by providing another form of relation among the parameters. For example, integrating the Eq. (8) by parts (from $t=0 \rightarrow T_{e}$ then $t=T_{e} \rightarrow T_{c}$ ) and using Eq. (4), one can easily find out the value of the $\beta$ as given below.

$$
\beta=\frac{E_{e}\left(\alpha^{2}+\omega_{z}^{2}\right)}{E_{o}\left\{e^{\alpha T_{e}}\left[\alpha \sin \left(\omega_{z} T_{e}\right)-\omega_{o} \cos \left(\omega_{z} T_{e}\right)\right]+\omega_{z}\right\}} .
$$

The above assumptions considered in the approximate LF model are relevant to the present work in the sense that, it provides a simple method to compute the parameters for characterizing the GFD that is comparable to that produced by the actual LF model (Qi \& Bi 1994). Further, the assumptions taken do not affect the speaker information present in the estimated parameters much. For example, the first assumption considers that $\beta T_{a}=1$, so that $T_{a}$ is relatively less (10\% of the glottal cycle duration) compared to $T_{e}$ and $T_{c}$. Since, $T_{a}$ is the derivative of the glottal flow at minimum of its first derivative, it does not have any apparent physical correspondence with human voicing events (Qi \& Bi 1994). Similarly, in the second assumption the minimum air flow during the end of each glottal cycle may not affect much because of the differentiation.

The parameters like $T_{o}, T_{e}, T_{c}, E_{e}$ are computed from the LP residual by locating the glottal cycles. The parameters like $\omega_{o}$ are $E_{o}$ are initially approximated and then $\alpha$ and $\beta$ are computed using Eqs. (4) and (9). These parameters are modified iteratively until the Eq. 7 is satisfied. To limit the computation, the process is bounded by 10 iterations. The number of iterations is chosen based on the observations from the experimental studies done in this work. It should be noted here that, unlike the earlier approach, this iteration process involves only computation of two parameters and adjust all parameters concurrently. Thus, the computational complexity and risk of unrealistic conditions are relatively less in the proposed approach.

2.2a Computation of $T_{o}, T_{e}, E_{e}$ and $T_{c}$ : Due to weak excitation, direct estimation of the glottal opening instant is a difficult task (Naylor et al 2007). Thus, in this work, first we compute the closing instant and then the corresponding opening instant is identified as the fixed fraction of the glottal cycle as suggested in Naylor et al (2007). Each glottal pulse is considered as the segment of the LP residual from respective glottal opening to closing instant. $T_{e}$ and $E_{e}$ are calculated by identifying the peak in the glottal pulse. The accuracy in the computation of all these parameters depends on how accurate the GCIs are estimated. There are several methods that have been proposed in the literature for computing the glottal closure instants and the most recently proposed zero-frequency filtering approach is found to be more accurate (Yegnenarayana \& Murthy 2009; Murthy \& Yegnanarayana 2008). The advantage of using this method is that it computes the glottal closure instants directly from the speech signal and does not require finding the closed phase region. A brief description of this method is given below (Yegnenarayana \& Murthy 2009; Murthy \& Yegnanarayana 2008).

The zero-frequency filtering method locates the GCIs by passing the speech signal through a zero-frequency resonator twice. The zero-frequency resonator is a second order infinite impulse response (IIR) filter located at $0 \mathrm{~Hz}$ (Murthy \& Yegnanarayana 2009). The purpose of passing the speech signal twice is to reduce the effects of all (high frequency) resonances (Murthy \& Yegnanarayana 2008). Passing the speech signal twice through a zero-frequency resonator 
is equivalent to four times successive integration. This will result in a filtered output that grows/decays as a polynomial function of time. The trend in the filtered signal is removed by subtracting the local mean computed over an interval corresponding to the average pitch period. The resulting mean subtracted signal is called as zero-frequency filtered signal (ZFFS). The positive zero crossings in the ZFFS correspond to the locations of GCIs (Murthy \& Yegnanarayana 2008). The steps involved in processing the speech signal to derive the ZFFS are given below.

(1) Difference the speech signal $s(n)$

$$
x(n)=s(n)-s(n-1) .
$$

(2) Pass the difference speech signal $x(n)$ twice through zero-frequency resonator

$$
y_{1}(n)=-\sum_{k=1}^{2} a_{k} y_{1}(n-k)+x(n)
$$

and,

$$
y_{2}(n)=-\sum_{k=1}^{2} a_{k} y_{2}(n-k)+y_{1}(n),
$$

where, $a_{1}=-4, a_{2}=6, a_{3}=-4$ and $a_{4}=1$.

(3) Compute the average pitch period using the autocorrelation over a $20 \mathrm{~ms}$ speech segment

(4) Remove the trend in $y_{2}(n)$ by subtracting the mean computed over average pitch period. The resulting signal

$$
y(n)=y_{2}(n)-\frac{1}{2 N+1} \sum_{m=-N}^{N} y_{2}(n+m),
$$

is the ZFFS. Here, $2 N+1$ corresponds to the number of samples in the window used for mean substraction.

The zero-frequency resonator filters out a mono-component centered around the 0 frequency from the speech signal. The present work considers the telephone speech data for the evaluation of the effectiveness of the proposed approach. In case of the telephonic speech, the frequency components below $300 \mathrm{~Hz}$ are heavily damped. The output of the zero-frequency resonator obtained from processing the telephonic speech may not give correct estimation of the GCIs. In Pati \& Prasanna (2011) and Yegnanarayana \& Prasanna (2010), it was suggested that, due to impulse-like nature of the LP residual, the pitch excitation information will spread across all the frequencies including the zero frequency and the positive zero-crossings in the ZFFS derived from the Hilbert envelope (HE) of the LP residual can be used to locate the GCIs accurately for telephonic speech. The HE is defined as the magnitude of complex time function of the LP residual (Yegnanarayana \& Prasanna 2010; Cohen 1995). The purpose of using the HE is to emphasize the peaks around the GCIs in each glottal cycle and hence the reinforcement of energy around the impulse at zero frequency (Ananthapadmanabha \& Yegnanarayana 1979; Yegnanarayana \& Prasanna 2010). Thus, the HE of the LP residual is used as an input to locate the GCIs.

Once the GCIs are computed, then the GOIs are computed as the following GCIs plus a fixed duration of the larynx cycle (Naylor et al 2007). The larynx cycle is considered as the minimum of difference between preceding and following GCI and the average pitch period. Closed-phase intervals have been reported to be $30 \%$ to $45 \%$ of the larynx cycle for normal speech. In this 
work, the opening instant of a glottal cycle is computed as the closing instant of the just preceding glottal cycle plus 30\% (minimum range) of the larynx cycle (Naylor et al 2007). It should be noted here that, since in the computation of the opening instant, both preceding and following glottal cycles closing instants are involved, the first and the last glottal cycles are ignored. Further, the glottal cycles duration may be beyond the practical range, that is of duration 5 to $20 \mathrm{~ms}$ (Deller et al 1993). This is because the successive glottal cycles may not be continuous. We consider only those glottal cycles that have 5 to $20 \mathrm{~ms}$ duration. The detailed procedure to compute the GOIs from GCIs are given below (Naylor et al 2007).

(1) Compute $P_{g}$, that is the pitch period of the $g^{\text {th }}$ glottal cycle as, $P_{g}=T_{c(g)}-T_{c(g-1)}$, where, $T_{c(g)}$ and $T_{c(g-1)}$ are the closing instants of the $g^{t h}$ and its just previous glottal cycle.

(2) Compute the average pitch period $\widehat{P}$, that is the maximum of seventh order median filtering of $P_{g}$ (Naylor et al 2007).

(3) Compute $T_{o(g+1)}$, that is the opening instant of the $(g+1)^{t h}$ glottal cycle using Eq. (14). The opening instants are considered as the $30 \%$ of the larynx cycles (Naylor et al 2007).

$$
T_{o(g+1)}=T_{c g}+0.03 \times \min \left[T_{c(g+1)}-T_{c(g)}, \widehat{P}\right] .
$$

(4) Consider only corresponding opening and closing instants those have the difference within 5 to $20 \mathrm{~ms}$ range.

A segment of voiced speech, ZFFS and the LP residual are shown in figures $4 \mathrm{a}-\mathrm{c}$, respectively. The positive zero-crossings in the ZFFS indicate the GCIs. The corresponding instants in the LP residual marked as ' $\mathrm{X}$ ' are considered as the end points of the LP residual blocks. The computed GOIs marked as ' $\mathrm{O}$ ' are considered as the starting points of the LP residual blocks. The locations

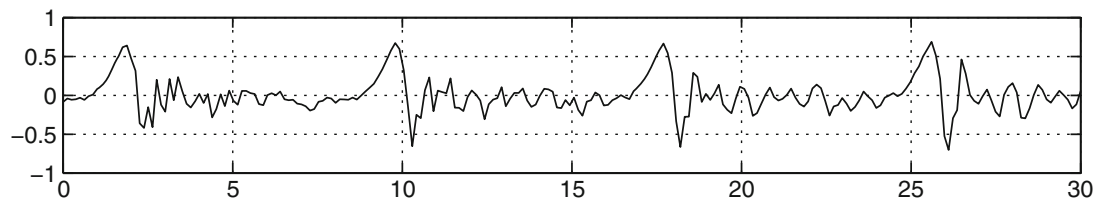

(a)

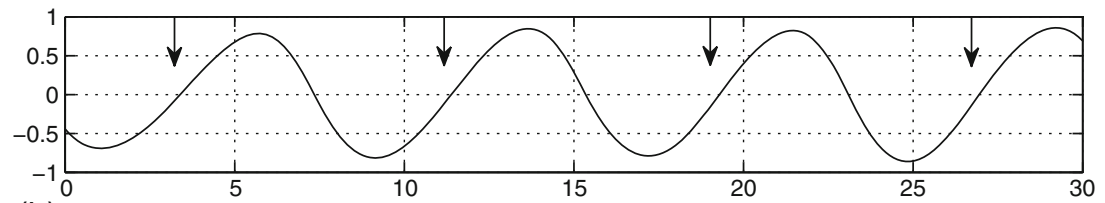

(b)

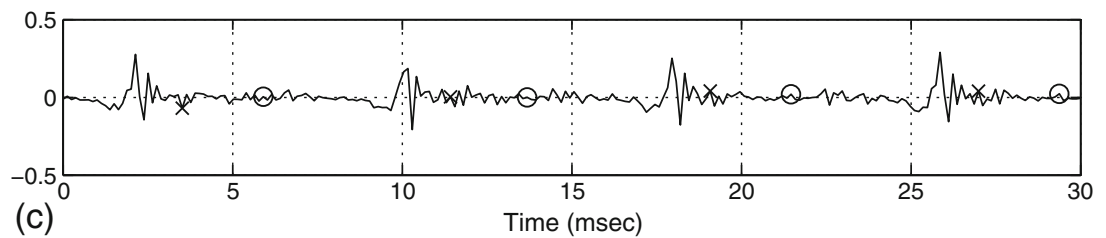

Figure 4. Estimation of the glottal cycles from the LP residual of the speech signal. (a) Speech signal. (b) ZFFS derived from the HE of the LP residual shown in (c). The location of the positive zero-crossings in the filtered signal (b) are shown by arrows. The 'Xs' and 'Os' in the LP residual (c) represent glottal closing and opening instants, respectively. 
and peaks of the LP residual blocks indicate their corresponding $T_{e}$ and $E_{e}$, respectively. It should be noted here that, the LP residual is an error signal resulting from the prediction of the speech signal (Makhoul 1975). The peaks in the LP residual corresponds to large error which may either be positive or negative. Thus, in finding the $T_{e}$ and $E_{e}$, we consider the absolute peaks in the LP residual blocks.

2.2b Computation of $T_{z}, E_{o}, \alpha$ and $\beta$ : Once the values of $T_{e}$ and $E_{e}$ are computed as described in section 2.2a, one can observe from the Eqs. (4) and 9 that for computation of the $\alpha$ and $\beta$, the only unknown parameters left are $T_{z}$ and $E_{o}$. Since the LP residual is an error signal it is difficult to find the first zero-crossings $T_{z}$ and then in turn the $E_{o}$ also, accurately. Thus, we propose to estimate these parameters through an iterative process that involves initial approximation of the parameters followed by modifications. The detailed procedure of the proposed approach is described below.

The parameters $T_{z}$ and $E_{o}$ are associated with the open-phase of the glottal cycle which is generally larger than the return phase. Thus, initially we assume that $T_{z}$ is $50 \%$ of the glottal cycle duration. With this assumption, $E_{o}$ is measured as the absolute maximum of the glottal cycle up to $T_{z}$. The reason for using the absolute maximum value is as mentioned earlier. Now, one can easily compute the value of $\alpha$ and $\beta$ from Eqs. (4) and (9), respectively. By observing these equations one can found that the parameter $\beta$ depends upon $\alpha$ and $\omega_{z}$. Thus to verify the accuracy of the initial estimation of the parameters, we prefer to use the constraint imposed by Eq.(7). Any modification in the value of $T_{z}$ will concurrently adjust all the remaining parameters. In every modification, the $T_{z}$ value is increased by $5 \%$ of the glottal cycle. The reason for increasing the $T_{z}$ value is due to the larger duration of the open phase. The steps involved for computing the LF parameters for each glottal cycle are summarized below.

(1) Initially, assume that $T_{z}=0.5 T_{c}$

(2) Compute, $w_{z}=\frac{\pi}{T_{z}}$

(3) Compute, $E_{o}$ as the maximum absolute of the sample values up to $T_{z}$

(4) Compute, $\alpha$ using Eq. (4)

(5) Compute, $\beta$ using Eq. (9)

(6) Compute, the value of $\beta T_{a}$

(7) If $\beta T_{a} \neq 1$, then replace $T_{z}=T_{z}+0.05 T_{c}$ and repeat from step 1

(8) Continue step 7 until $\beta T_{a} \simeq 1$ or the number of repetitions is 10 .

It should be noted here that an iterative method was previously applied to estimate the LF model parameters in Plumpe et al (1999) and Iseli \& Alwan (2000). In this method, the iteration process minimizes the error between the estimated glottal flow and the LF model. The error in the estimated glottal flow mostly due to the requirement of the closed-phase region estimation which may affect the computational accuracy of the parameters. The proposed approach does not require the estimation of the closed-phase region. This approach also adjusts all parameters concurrently and hence provides global modification in estimating the parameters. Thus, it is expected that the proposed approach may be effective in computation of the LF parameters for explicit modelling of the subsegmental excitation information.

\subsection{Modelling subsegmental excitation information from LF parameters}

The LF parameters are derived from the respective LP residual of the speech signal and hence they represent the GFD characteristics of the individual speaker. Both static and dynamic nature of the LF parameters may be used to capture the speaker information. Modelling speaker 
information by LF parameters may be viewed as the explicit approach. We use the proposed approach to compute the LF parameters for modelling the subsegmental excitation information explicitly and demonstrate its significance by speaker identification and verification studies.

2.3a Speaker-specific feature from LF parameters: The seven LF parameters described in the table 1 may be classified into two groups as: wave shaping and timing parameters. The wave shaping parameters that include $\omega_{o}, \alpha$ and $\beta$ characterize the shape of the GFD. The timing parameters that include $T_{o}, T_{e}$ and $T_{c}$ characterize the glottal timing information. The wave shaping parameters are directly used for feature representation. The timing parameters are first normalized with respect to the length of the glottal cycle and then used for feature representation. The normalization is performed to limit the values of the absolute times. Otherwise, these absolute time values will increase beyond limit with increase in the number of the glottal cycles (Plumpe et al 1999).

The discrete domain representation of the four wave shaping parameters are given by

$$
\begin{aligned}
\Omega_{z} & =\frac{\pi}{N_{z}} \\
\alpha & =\frac{1}{N_{e}} \ln \left[-\frac{E_{e}}{E_{o} \sin \left(\Omega_{z} N_{e}\right)}\right] \\
\beta & =\frac{E_{e}\left(\alpha^{2}+\Omega_{z}^{2}\right)}{E_{o}\left\{e^{\alpha N_{e}}\left[\alpha \sin \left(\Omega_{z} N_{e}\right)-\Omega_{o} \cos \left(\Omega_{z} N_{e}\right)\right]+\Omega_{z}\right\}},
\end{aligned}
$$

where, $N_{z}$ and $\Omega_{z}$ are sample-time counterparts to their corresponding continuous time variables $T_{z}$ and $\omega o$, respectively.

The discrete domain representation of the three normalized timing parameters are given by Plumpe et al (1999)

$$
\begin{aligned}
\text { Close quotient (CQ) } & =\frac{N_{o}-N_{c-1}}{N_{e}-N_{e-1}} \\
\text { Open quotient (OQ) } & =\frac{N_{e}-N_{o}}{N_{e}-N_{e-1}} \\
\text { Return quotient (RQ) } & =\frac{N_{c}-N_{e}}{N_{e}-N_{e-1}},
\end{aligned}
$$

where, $N_{o}, N_{e}$ and $N_{c}$ are sample-time counterparts to their corresponding continuous time variables $T_{o}, T_{e}$ and $T_{c}$, respectively.

The speaker-specific nature of the wave shaping parameters and the normalized timing parameters is demonstrated by comparing their histograms from two different female speakers ( $F S-1$ and FS-2) shown in figure 5. The histograms are obtained from about $30 \mathrm{~s}$ of data of each speaker. The text of the speech and the gender of the speakers remain same for fair comparison. It can be observed from the histograms that there is a separation in the distribution of the parameters value across speakers. In general, the wave shaping parameters are less speaker-specific than the timing parameters. In particular, the distribution of the OQ and RQ values are significantly different. This may be due to the perceptual importance of the open and return phase that determines the spectral tilt (Veldhuish 1998; Strik 1998; Qi \& Bi 1994).

The wave shaping and timing parameters computed from a glottal cycles are concatenated to form the feature vector. The value of the wave shape parameters are varying across the different range. For example, the timing parameters are within the range of 0 to 1 , where as, the value of 

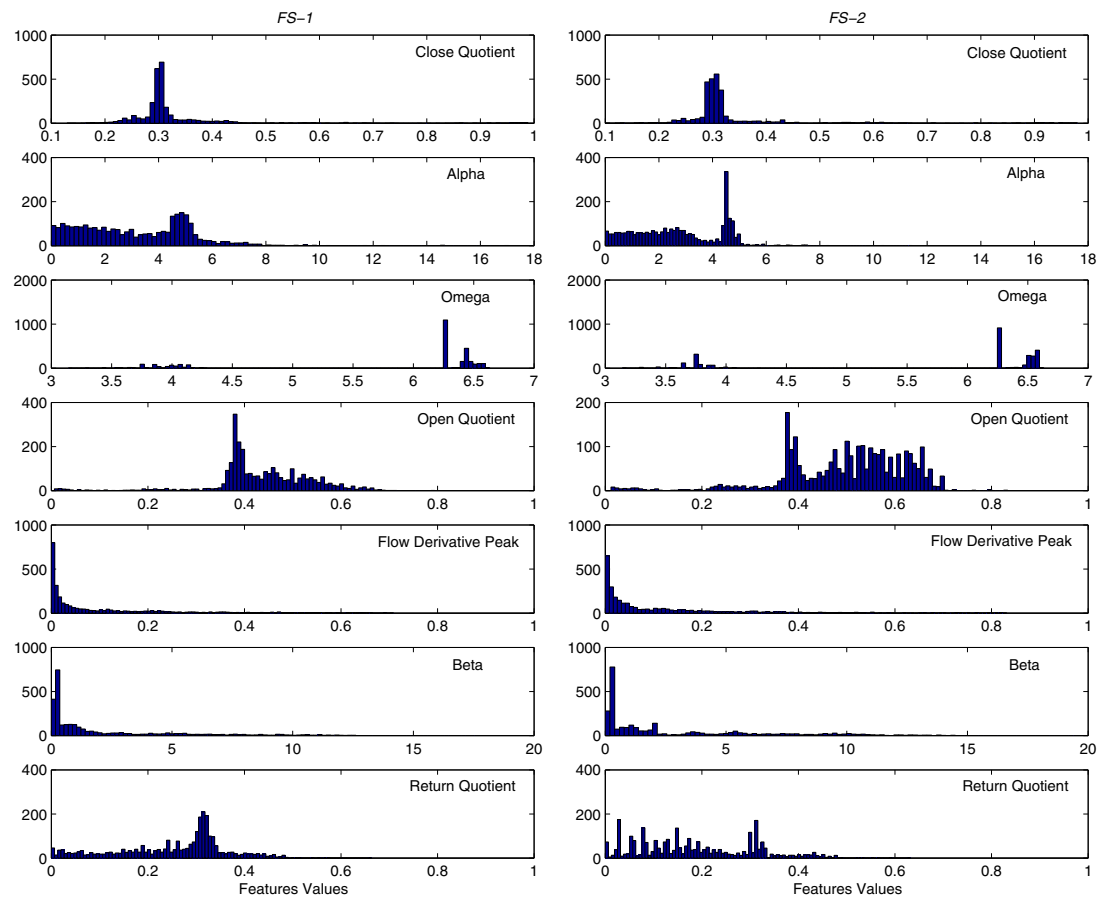

Figure 5. Comparison of the histograms of seven components of the glottal flow feature for two different female speakers. The feature component values are divided across 100 histogram bins.

$\alpha$ and $\beta$ are in the range of the $0-20$ and the value of the $\Omega_{z}$ in the range of 3.5-7. Therefore, to avoid the large variations, the computed parameters need to be normalized to a common range. In this work, we normalize these parameters by zero-mean unit variance and then used to represent the features for building the speaker models and later for testing. In this work, features from the LF parameters are called as $G F D$ features.

2.3b Speaker-specific feature from dynamics of LF parameters: The fine variations of the GFD like aspiration and ripple also provide useful speaker information (Plumpe et al 1999). This information was captured through formant modulation in the closed phase region and used for speaker identification study (Plumpe et al 1999). It was shown that, the recognition performance of the features associated with the fine variations of the glottal flow is relatively poor but provide additional information to the $G F D$ feature. Motivated by this we try to use this information in our study. However, due to the difficulty in finding the closed phase region, we exploit the dynamic nature of the LF parameters to capture the fine variations. The hypothesis is that, the variation in the LF parameters from one glottal cycle to the other may be attributed to the fine variations in the glottal cycles. Figure 6 shows the contours of the LF parameters obtained from same segment of the speech for FS-1 and FS-2. Due to difference in the pitch, the number of LF parameters obtained are different across speakers. Thus, cycle-to-cycle comparison of the LF parameters may not be fair. The variation in the parameters value from one cycle to the next cycle is different across speakers. These differences may be due to the different speaking style that affects the glottal cycles. 

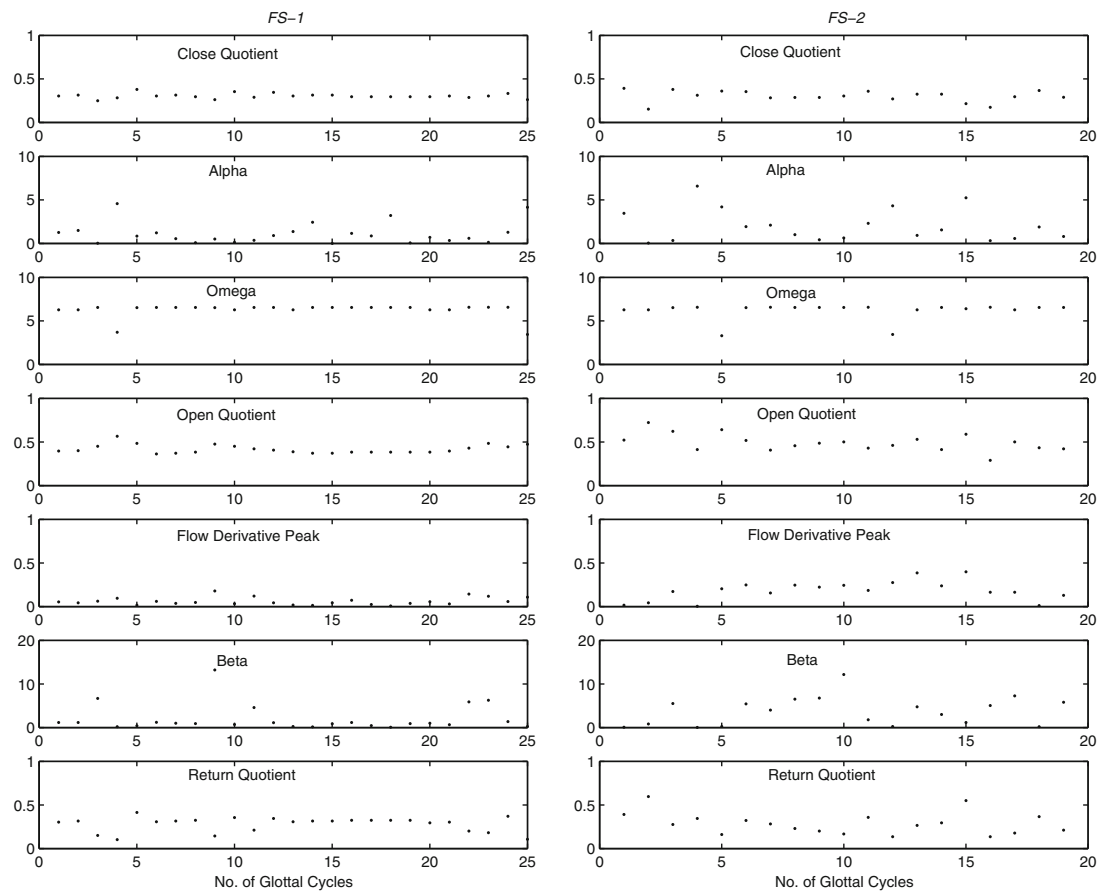

Figure 6. Example of the contours of seven components of the $G F D$ feature from $0.5 \mathrm{~s}$ duration of speech for two different female speakers.

In this work, the speaker-specific information associated with the dynamics of the LF parameters are represented by standard delta $(\Delta)$ and delta delta $(\Delta \Delta)$ measures (Furui 1981a; Reynolds \& Rose 1995). The commonly used equations for $\Delta$ and $\Delta \Delta$ measures are given by Bimbot et al (2004) and Furui (1981b)

$$
\begin{gathered}
\Delta X_{m}(n)=\frac{\sum_{i=-T}^{T} k_{i} X_{m}(n+i)}{\sum_{i=-T}^{T}|i|} \\
\Delta \Delta X_{m}(n)=\frac{\sum_{i=-T}^{T} k_{i} \Delta X_{m}(n+i)}{\sum_{i=-T}^{T}|i|},
\end{gathered}
$$

where $X_{m}(n)$ represents the $m^{\text {th }}$ component of the feature $X$ for the $n^{\text {th }}$ frame, $k_{i}$ is the $i^{\text {th }}$ weight and $T$ is the number of successive frames. Generally $T$ is considered as 2 . The $\Delta$ and $\Delta \Delta$ measures give the rate of change of the LF parameters values from cycle to cycle. Since, the LF parameters are unique, it is expected that their $\Delta$ and $\Delta \Delta$ measures may also contribute speaker information. The $\Delta$ and $\Delta \Delta$ values are concatenated with LF parameters and together used as the features. We call them as $G F D_{\Delta-\Delta \Delta}$ feature. 


\subsection{Speaker recognition studies using $G F D$ and $G F D_{\Delta-\Delta \Delta}$ features}

2.4a Experimental set-up: In this work for speaker recognition studies, we conduct both identification and verification tasks on text-independent mode that refers to no constraint in the use of text of speech for building the models and later for comparison. The identification task involves in finding the most likely speaker and verification task validates the identity claim. For identification experiment GMM approach is used to build the speaker models and decision is taken based on the log-likelihood score (Reynolds \& Rose 1995). The speaker of the model having highest log-likelihood score is considered as the identified speaker. The performance is expressed in terms of identification accuracy expressed in percentage. The identification experiment is conducted on two separate data sets, called as Set-1 and Set-2. The speech data for Set-1 and Set-2 are collected from NIST-99 and NIST-03 databases, respectively (Przybocky \& Martin 2000, 2003). NIST-99 is used as the representation of clean data collected over land-line and NIST-03 as relatively noisy data, since it is collected over mobile phones. The speakers having matched condition and testing data of at least $30 \mathrm{~s}$ are considered. Each set consists of 90 speakers that includes 48 males and 42 females.

In case of verification task, state-of-the-art GMM-universal back ground (GMM-UBM) approach is used for speaker modelling. The UBM is built from approximately forty hours speech data of 200 speakers (100 males and 100 females from switchboard database) and serves as the imposter models. The gaussian mixture speaker models are built by adaption of UBM. Only the means are adapted and the weights and variances of the speaker models and the UBM remain same. The verification is made based on the log-likelihood (LLR) score. For a given test utterance, the LLR is given by

$$
L L R=\log P\left(s \lambda_{c}\right)-\log P\left(s \lambda_{u}\right),
$$

where, $P\left(s \lambda_{c}\right)$ and $P\left(s \lambda_{u}\right)$ are the likelihoods given by the claimed speaker model and the UBM, respectively. The performance is given by detection error tradeoff (DET) curve based on genuine and imposter LLRs (Martin et al 1997). From the DET curve, equal error rate (EER) is found by choosing a threshold such that false acceptance rate (FAR) is equal to false rejection rate (FRR). EER is expressed in percentage.

The speaker verification experiment is conducted on the whole NIST-03 database (Przybocky \& Martin 2003). The database consists of 356 target speakers. Each speaker has a training data of about 2 min duration, which is used to build the models. There are totally 2559 test utterances with duration of 15-45 s. There are totally 2559 test utterances with duration of 15-45 s. Each test utterance is tested against 11 hypothesized speakers that include the genuine speaker and ten imposters. Each test is performed on two cases. The first case refers to Clean test, where the test signal is directly used for the verification. The second case refers to Noisy test, where we add the white noise with signal-to-noise ratio (SNR) around $9 \mathrm{~dB}$ to the test signal and then used for the verification. The objective of doing the Noisy test is to verify the robustness of the features against noise for speaker verification task. In both cases the experimental conditions remain same for fair comparison.

2.4b Divergence measure: To verify the discriminating ability of the features, we use Divergence measure which is a generalization concept of the F-ratio to the multidimensional case (Pruzansky \& Mathews 1964; Atal 1976; Duda \& Hart 2001; Haeb-Umbach 1999). Divergence is a statistical measure of effectiveness of a set of distributions for discriminating between categories (Atal 1976). It is measured from two covariance matrices: Between class and Within class covariance matrices (Duda \& Hart 2001). For our application, the Divergence of a feature 
as a measure of speaker discriminating ability can be measured from intra-speaker $(W)$ and interspeaker (B) covariance matrices (Atal 1976; Haeb-Umbach 1999). W represents the variation of the feature vectors within an individual speaker and $B$ represents the variation of the feature vectors between different speakers. A feature is said to be more discriminating, if it is less variant within an individual speaker and more variant between different speakers (Wolf 1972). The scalar measure of this property is the trace of the matrix $W^{-1} B$ called as Divergence (Atal 1976; Haeb-Umbach 1999). The trace of a matrix is the sum of its diagonal elements. Let us consider a distribution $\chi$ consists of set of feature vectors of $S$ speakers. Each $s^{\text {th }}$ speaker, where $s=1,2, \ldots, S$, in the distribution has $n_{s}$ number of $d$-dimensional feature vectors. If $x_{\psi_{s}}$ is the $\psi_{t h}$ feature vector of the $s^{t h}$ speaker then its mean feature vector is given by

$$
m_{s}=\frac{1}{n_{s}} \sum_{\psi_{s}=1}^{n_{s}} x_{\psi_{s}}, \quad x_{\psi_{s}} \in n_{s} .
$$

There is a total of $N_{S}=\sum_{S=1}^{S} n_{S}$ number of such mean feature vectors in the distribution $\chi$. The total mean feature vector of the distribution $\chi$ is given by Duda \& Hart (2001) and Haeb-Umbach (1999)

$$
m=\frac{1}{N_{S}} \sum_{s=1}^{S} n_{s} m_{s} .
$$

Then the covariance matrices $W$ and $B$ are given by Haeb-Umbach (1999)

$$
\begin{gathered}
W=\sum_{s=1}^{S} \sum_{\psi_{s}=1}^{n_{s}}\left(x_{\psi_{s}}-m_{S}\right)\left(x_{\psi_{s}}-m_{S}\right)^{T} . \\
B=\sum_{s=1}^{S} n_{s}\left(m_{s}-m\right)\left(m_{s}-m\right)^{T},
\end{gathered}
$$

where $T$ represents the transpose of a vector. A feature with higher Divergence value usually provides more discriminatory information.

The Divergence values of the $G F D$ and $G F D_{\Delta-\Delta \Delta}$ features given in the table 2 indicate that the LF parameters computed by the proposed approach have the speaker discriminating ability. By comparing their respective Divergence values it can be observed that the $G F D_{\Delta-\Delta \Delta}$

Table 2. Divergence and speaker identification performance (\%)

\begin{tabular}{|c|c|c|c|c|c|c|}
\hline \multirow{2}{*}{$\begin{array}{l}\text { Feature } \\
\text { database }\end{array}$} & \multicolumn{2}{|c|}{$G F D$} & \multicolumn{2}{|c|}{$G F D_{\Delta-\Delta \Delta}$} & \multicolumn{2}{|c|}{$S u b$} \\
\hline & Div & Perf & Div & Perf & Div & Perf \\
\hline Set-1 & 48.74 & 26 & 111.87 & 30 & 13.03 & 64 \\
\hline Set-2 & 34.28 & 20 & 89.58 & 25 & 9.75 & 57 \\
\hline$R D$ & 30 & 23 & 20 & 17 & 25 & 11 \\
\hline
\end{tabular}
of subsegmental excitation information from explicit and implicit modelling approaches. Div, Perf and $R D$ represent Divergence, performance and relative degradation, respectively. 

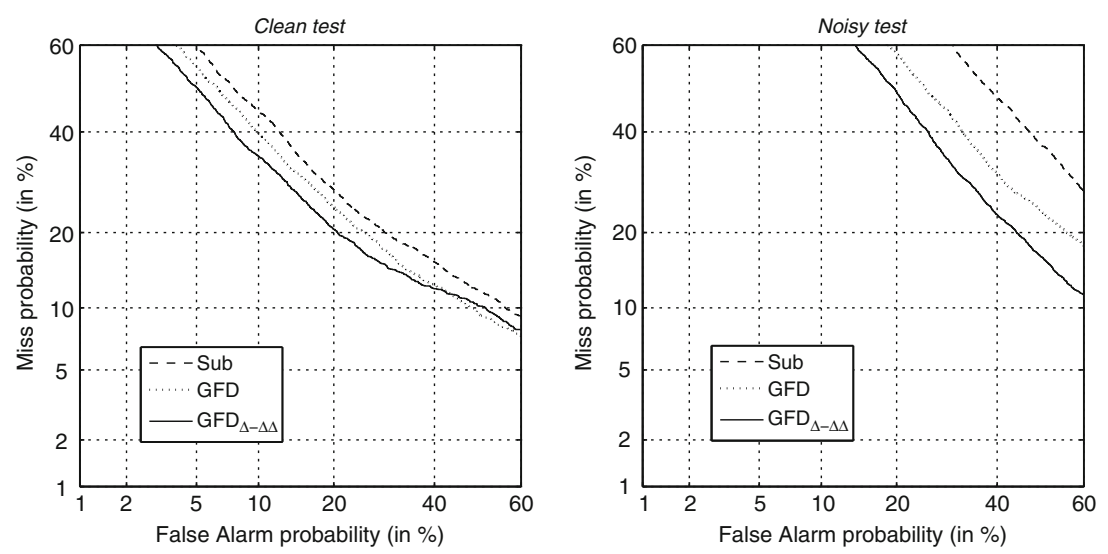

Figure 7. DET curves from the speaker verification experiments using evidence from explicit and implicit modelling of the subsegmental excitation information.

features have significantly better speaker discriminating ability. This shows that the speaker discriminating ability of the LF parameters is increased by incorporating their dynamic values. Further, due to noise, the relative degradation in the discriminating ability of the $G F D_{\Delta-\Delta \Delta}$ feature is relatively less. For example, in case of $G F D_{\Delta-\Delta \Delta}$ feature, the relative degradation in the Divergence value is $20 \%$, as against $20 \%$ for $G F D$ feature. These observations indicate that the $G F D_{\Delta-\Delta \Delta}$ features have relatively more speaker discriminating ability and robust against noise. It is expected that the LF parameters together with their dynamic values may provide good recognition accuracy.

2.4c Results and discussion: The speaker identification results of $G F D$ and $G F D_{\Delta-\Delta \Delta}$ features are given in table 2. The DET curves from the speaker verification results of the $G F D$ and $G F D_{\Delta-\Delta \Delta}$ features are shown in figure 7 and their corresponding EER are given in the table 3 . The recognition results show that explicit modelling of the subsegmental excitation information by the LF parameters computed using the proposed approach is useful for speaker recognition task. The speaker identification and verification performances achieved by $G F D_{\Delta-\Delta \Delta}$ feature is relatively better than the GFD feature. For example, for Set-1 and Set-2 data sets, the identification performances achieved by $G F D_{\Delta-\Delta \Delta}$ feature are $30 \%$ and $25 \%$ as against $26 \%$ and $20 \%$ in case of $G F D$ feature, respectively. For Clean and Noisy test cases, the EER achieved by $G F D$ and $G F D_{\Delta-\Delta \Delta}$ features are around $20.28 \%$ and $31.30 \%$ as against $22.32 \%$ and $35.68 \%$ in case of $G F D$ feature, respectively. Further, the recognition performance of $G F D$ and $G F D_{\Delta-\Delta \Delta}$

Table 3. Speaker verification performance (\%) of subsegmental excitation information from explicit and implicit modelling approaches.

\begin{tabular}{lccc}
\hline Feature test & $G F D$ & $G F D_{\Delta-\Delta \Delta}$ & Sub \\
\hline Clean & 22.32 & 20.28 & 23.75 \\
Noisy & 35.68 & 31.31 & 43.76 \\
RD & 60 & 54 & 84 \\
\hline
\end{tabular}


features degrades due to noise. But, the degradation in case of $G F D_{\Delta-\Delta \Delta}$ features is relatively less. For example, the relative degradation in the identification and verification performances of the $G F D_{\Delta-\Delta \Delta}$ features are $17 \%$ and $54 \%$, as against $23 \%$ and $60 \%$ in case of $G F D$ feature, respectively. This demonstrates the relative robustness of the $G F D_{\Delta-\Delta \Delta}$ features against noise. From these observations, we mention that the proposed approach of computing the LF parameters together with their dynamics may be the best possible way of modelling the subsegmental excitation information explicitly.

\section{Implicit modelling of subsegmental excitation information}

The large peaks approximately correspond to the negative peaks of the respective GFD cycles. The strength and location of these peaks are different across speakers. The variation in the sequence of samples around the epochs mostly represent the glottal activity information. Thus, the direct use of $5 \mathrm{~ms}$ (40 samples for $8 \mathrm{kHz}$ speech signal) blocks is found to be useful to capture the glottal activity information. It is not clearly understood what part of the subsegmental excitation information is captured in the LP residual blocks and hence corresponds to implicit modelling. The proposed explicit approach of modelling the subsegmental excitation information is relatively more compact and involves less computational complexity. For example, for a given two minute training data, the number of feature vectors available for implicit modelling is around 40000 as against to 10000 for explicit modelling. Computational complexity is less in the sense that, the zero-frequency approach used in the proposed approach for locating the GCIs can also be used for modelling the suprasegmental pitch and epoch strength contours information. It is expected that the proposed explicit approach of modelling the subsegmental excitation information may be more effective.

As mentioned in the introduction section, implicit modelling approach does not involve any parameterization. Thus, the excitation signal is directly processed in blocks to capture the subsegmental excitation information. With proper LP order (for example 8-20 in case of speech sampled at $8 \mathrm{kHz}$ ), the LP residual can be used as the best approximation of the excitation signal (Prasanna et al 2006). Thus, the LP residual is mostly used as the representation of the excitation signal (Prasanna et al 2006; Murty et al 2004; Yegnanarayana et al 2001; Murty \& Yegnanarayana 2006; Pati \& Prasanna 2008, 2011). The LP residual is an impulse-like signal with large peaks around the approximate location of the epochs (Ananthapadmanabha \& Yegnanarayana 1979; Murthy \& Yegnanarayana 2008). Most of the speaker-specific subsegmental level information is present around the epochs (Murty \& Yegnanarayana 2006). This includes the epoch strength and the temporal variation of the samples around the epochs. The information around the epochs may be attributed to the glottal cycle activities. For example, large peaks in the LP residual may correspond to the negative peak of the GFD. Hence, it is expected that the LP residual can be used directly to model the subsegmental level information implicitly.

\subsection{Modelling subsegmental excitation information from LP residual}

The speech and LP residual signals of two male speakers ( $M S$ - 1 and $M S-2)$ are shown in figures 8 $a$ and $b$, respectively. The text of the speech signals remain same for fair comparison. Like speech signals, the LP residual of the two speakers are different. In addition to the significant difference between their pitch and epoch strength, the temporal variations of the sequence of samples around the epochs are also different. For example, the average pitch period and epoch strength in case of $M S-1$ is relatively more than the $M S-2$. In case of $M S-1$, the amplitude of the 


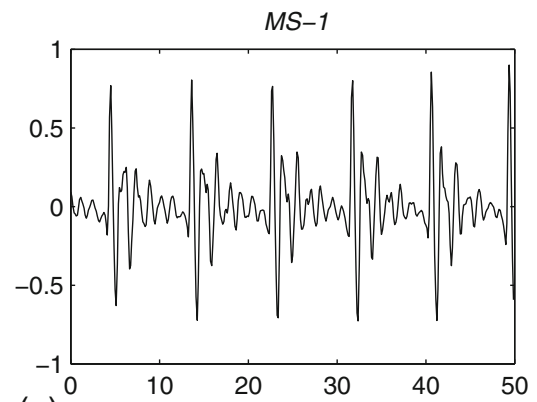

(a)

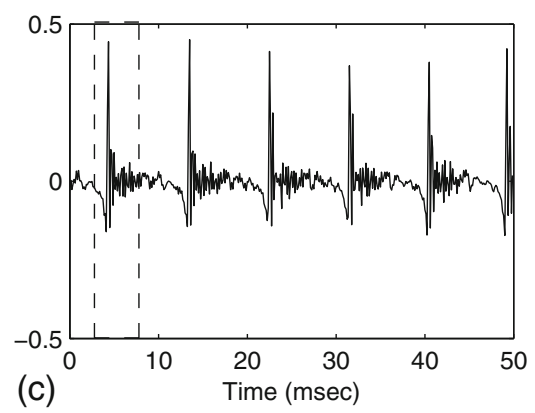

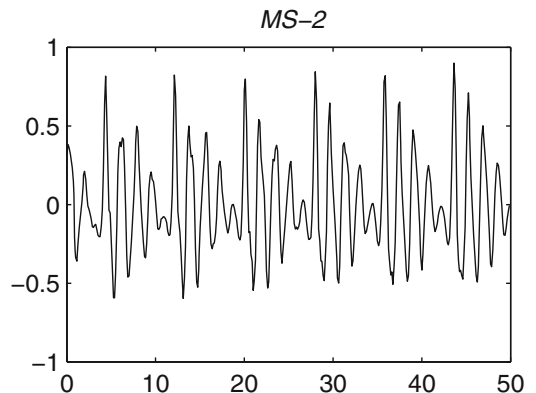

(b)

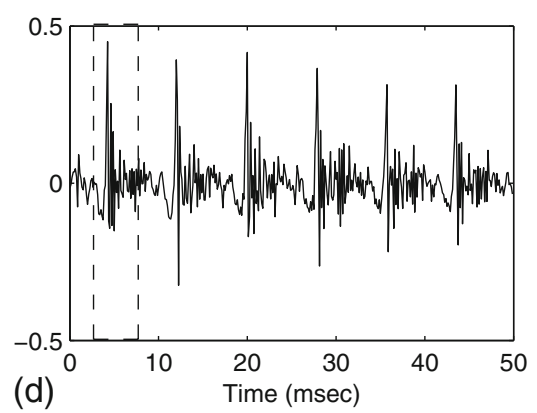

Figure 8. Example of speech and LP residual signals of two male speakers. (a) and (b) Speech signals. (c) and (d) LP residuals. The dotted box in (c) and (d) represents the LP residual block processed at the subsegmental level.

samples that give the sequence information of the glottal cycles is relatively smoother than $M S-2$. This indicates that, the sequence of LP residual samples around the epochs contains speakerspecific information.

The subsegmental information is mostly modelled by processing the excitation signal in blocks of 3-5 ms blocks. In this work, the LP residual is processed in blocks of $5 \mathrm{~ms}$ with $2.5 \mathrm{~ms}$ shift to represent the subsegmental level information as suggested in Pati \& Prasanna (2011). The higher range $(5 \mathrm{~ms})$ of the subsegmental level processing is considered for maximum representation of the glottal cycle information and $2.5 \mathrm{~ms}$ to get maximum number of feature vectors for better modelling. The dotted box in figures $8 \mathrm{c}$ and $\mathrm{d}$ show the nature of the LP residual block that is processed at the subsegmental level for $M S-1$ and $M S-2$, respectively. It can be observed that the nature of the sequence of samples of the subsegmental level block that represent the glottal cycle activity information is different across speakers. This indicates that the subsegmental level LP residual blocks can be used to model the glottal cycle activity information implicitly. It should be noted here that, the blocks are processed without any pitch synchronization. This is because, the objective is to capture the maximum information that is available in the LP residual. By pitch synchronization, most of the blocks may be discarded as unvoiced. Since, there is no parameterizations is involved, it may be difficult to capture the maximum information from the pitch synchronized blocks only. In this work, the subsegmental LP residual blocks used to model the speaker-specific information is called as $S u b$ feature.

The Divergence value of the $S u b$ features are given in the table 2. The Divergence value of $S u b$ features for Set-1 and Set-2 data sets are $13.03 \%$ and $9.75 \%$, respectively. Since the Divergence value of the $S u b$ features is larger than, they have large inter-speaker variability and small 
intra-speaker variability. This shows that the $S u b$ features may be useful for speaker recognition tasks. Like $G F D$ and $G F D_{\Delta-\Delta \Delta}$ features, the discriminating ability of the $S u b$ feature is also decreased for relatively more noisy speech data. For example, the relative decrement in the Divergence value from Set-1 to Set-2 is $25 \%$. In the following subsection the significance of the $S u b$ features is demonstrated by speaker identification and verification studies.

\subsection{Speaker recognition studies using sub features}

The speaker identification results of $S u b$ feature are given in the table 2. The DET curves from the speaker verification results of the $S u b$ features are shown in the figure 7 and their corresponding EER values are given in table 3. The speaker identification performance achieved by Sub feature for Set-1 and Set-2 data sets are 64\% and 57\%, respectively. For Clean and Noisy test cases, the EER achieved by $S u b$ features are around $23.75 \%$ and and $43.77 \%$, respectively. The recognition results show that the $5 \mathrm{~ms}$ LP residual blocks can be used for implicit modelling of the subsegmental excitation information for speaker recognition. In this case also, the performance of the $S u b$ features degrades due to noise. The relative degradation in the recognition performance of the $S u b$ feature for identification and verification task are $11 \%$ and $84 \%$, respectively. The degradation in the identification performance is significantly less than the verification task. In the next section a comparative study is made between the implicit and explicit approaches for modelling the subsegmental excitation information and demonstrate their significance for speaker recognition.

\section{Comparison of explicit and implicit modelling of subsegmental excitation information}

The study so far made in this paper demonstrates that the subsegmental excitation information can be modelled explicitly or implicitly for speaker recognition. For explicit modelling, the $G F D_{\Delta-\Delta \Delta}$ features derived from the LF parameters computed using the proposed approach can be used. For implicit modelling, the $S u b$ features derived from the overlapping LP residual blocks can be used. In this section, a comparative study is made between the explicit and implicit approaches for modelling the subsegmental excitation information for speaker recognition. The comparison is made based on computational complexity, speaker discriminating ability, recognition accuracy, in providing the different evidence to vocal tract features and robustness against noise.

\subsection{Nature of speaker-specific evidence in explicit and implicit modelling}

In implicit modelling, the $5 \mathrm{~ms}$ LP residual blocks are directly processed to model the subsegmental excitation information. On the other hand, for explicit modelling the characteristics of the GFD is parameterized from the LP residual blocks. Figure 9 shows the example of GFD cycles and the corresponding LP residual of two Males ( $M S-1$ and $M S-2)$ and one female $(F S-1)$ speakers taken from arctic database (Kominek \& Black 2004). The text of the speech signal in all cases remains same for fair comparison. The GFD cycles give a clear view of the glottal timing and the shape at different phases as well. These characteristics are parameterized by the wave shaping and timing LF parameters and hence corresponds to explicit modelling. Since, these parameters explicitly characterize the glottal flow, it is expected that the proposed approach may provide better recognition accuracy. On the other hand, due to noise-like nature, the glottal timing and shape at different phases are not clearly visible from the LP residual. Similar to glottal waves, the shape of LP residuals is also different across speakers and hence may be speaker-specific. 

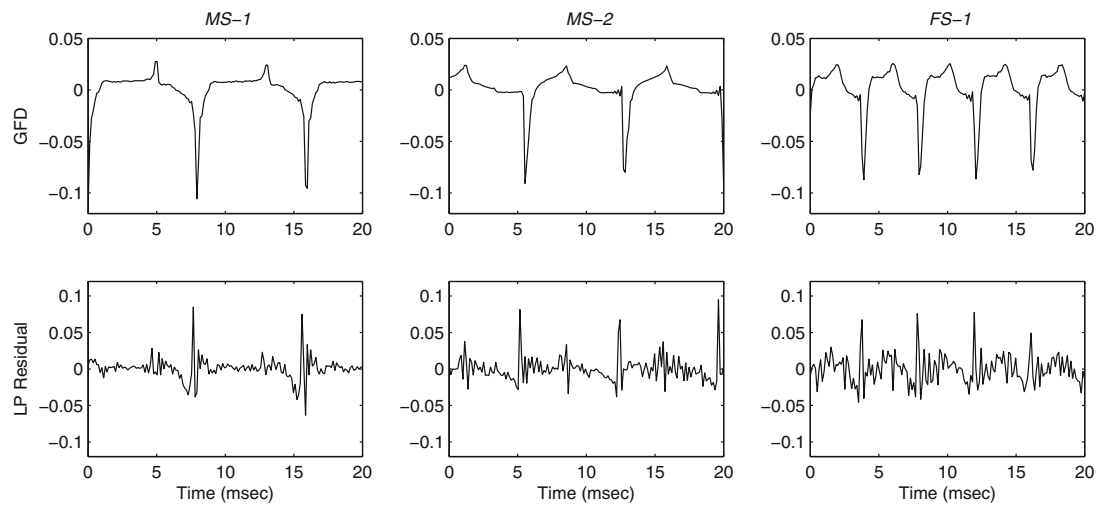

Figure 9. Example of GFD and LP residual segments of two males and one female speakers from a common utterance.

The large peaks approximately correspond to the negative peaks of the respective GFD cycles. The strength and location of these peaks are different across speakers. The variation in the sequence of samples around the epochs mostly represent the glottal activity information. Thus the direct use of $5 \mathrm{~ms}$ (40 samples for $8 \mathrm{kHz}$ speech signal) blocks is found to be useful to capture the glottal activity information. It is not clearly understood what part of the subsegmental excitation information is captured in the LP residual blocks and hence corresponds to implicit modelling. The proposed explicit approach of modelling the subsegmental excitation information is relatively more compact and involves less computational complexity. For example, for a given two minute training data, the number of feature vectors available for implicit modelling is around 40000 as against to 10000 for explicit modelling. Computational complexity is less in the sense that, the zero-frequency approach used in the proposed approach for locating the GCIs can also be used for modelling the suprasegmental pitch and epoch strength contours information. It is expected that the proposed explicit approach of modelling the subsegmental excitation information may be more effective.

\subsection{Significance of explicit and implicit modelling for speaker recognition}

The Divergence measure described in section $2.4 \mathrm{~b}$ can be used to verify the usefulness of the explicit and implicit approaches of modelling the subsegmental excitation information for speaker recognition. By comparing the Divergence value of $G F D_{\Delta-\Delta \Delta}$ and $S u b$ features given in the table 2, it can be observed that the explicit approach have in general more speaker discriminating ability. The Divergence value of both $G F D_{\Delta-\Delta \Delta}$ and $S u b$ features is decreased for more noisy database. The relative degradation in the Divergence value from Set-1 to Set-2 for $G F D_{\Delta-\Delta \Delta}$ feature is around $17 \%$, that is less than $23 \%$ as in case of the $S u b$ feature. These observation indicate that the proposed $G F D_{\Delta-\Delta \Delta}$ feature may be more effective in modelling the subsegmental excitation information for speaker recognition. However, $G F D_{\Delta-\Delta \Delta}$ feature may not be more effective for both speaker identification and verification tasks. Because, the Divergence takes into account the inter and intra variances and hence has no simple relation with recognition performance (Atal 1972). For example, higher Divergence value may be achieved either with low intra-variation or high inter-variation. In case of verification task for a genuine trail, where one-to-one comparison is made, the low intra-variation than more inter-variation may 
be preferred. In this case a feature with higher Divergence value due to high inter-variation may not be useful. On the other hand, in case of text independent identification task, where one-tomany comparisons are made, the more inter-variation than low intra-variation may be preferred. In this case a feature with higher Divergence value due to low intra-variation may not be useful. To measure qualitatively the usefulness of the $G F D_{\Delta-\Delta \Delta}$ and $S u b$ features for speaker identification and verification tasks, the trace of their respective inter and intra covariance matrices $(B$ and $W$ ) can be used as the independent scalar measures (proportional) of the inter and intra variances, respectively.

The measurement of the inter and intra variations depends on the dimension of the feature vectors. The dimension of the $G F D_{\Delta-\Delta \Delta}$ and $S u b$ features are different, for example 21 and 40, respectively. For fair comparison, the inter and intra variances need to be normalized. In this work, we normalize the inter and intra variations by their Divergence value and used as the assessment measure to verify the usefulness of the explicit and implicit approaches of modelling the subsegmental excitation information for speaker identification and verification tasks.

The inter and intra variances values of $G F D_{\Delta-\Delta \Delta}$ and $S u b$ features are given in the table 4. It can be observed from this table that in general (for both clean and noisy speech) the $G F D_{\Delta-\Delta \Delta}$ features have relatively less inter and intra variance than the Sub features. The higher Divergence value in case of $G F D_{\Delta-\Delta \Delta}$ feature may be due to low intra-variance. On the other hand, the lower Divergence value in case of $S u b$ feature may be due to higher intra-variance. These observations indicate that the proposed explicit approach of modelling the subsegmental excitation information may be more effective for speaker verification task. Similarly, the implicit modelling of the subsegmental excitation information by the $5 \mathrm{~ms}$ LP residual blocks may be more effective for speaker identification task. This in fact, we can observe by comparing the speaker identification and verification results of $G F D_{\Delta-\Delta \Delta}$ and $S u b$ features from the tables 2 and 3. The $G F D_{\Delta-\Delta \Delta}$ and $S u b$ features provide better performance for speaker verification and identification tasks, respectively.

The robustness of the speaker-specific evidence present in explicit and implicit modelling of the subsegmental excitation information can be demonstrated by comparing the amount of the relative degradation in the recognition performance due to noise by $G F D_{\Delta-\Delta \Delta}$ and $S u b$ features from the tables 2 and 3. The performance of both the $G F D_{\Delta-\Delta \Delta}$ and $S u b$ features is affected by noise. In case of the identification task, the degradation in the $G F D_{\Delta-\Delta \Delta}$ performance is of $23 \%$, which is more than the $S u b$ feature of around $11 \%$. On the other hand, the degradation in the verification performance of the $G F D_{\Delta-\Delta \Delta}$ feature is $54 \%$, which is less than the Sub feature of around $84 \%$.

From the above study, it is observed that the speaker-specific evidence present in the $G F D_{\Delta-\Delta \Delta}$ features is relatively more effective and robust for speaker verification task. The speaker-specific evidence present in the $S u b$ features is relatively more effective and robust for speaker identification task. It is suggested that to gain maximum benefit from the subsegmental

Table 4. Inter-variance and intra-variance values of $G F D_{\Delta-\Delta \Delta}$ and $s u b$ features from Set-1 and Set-2 data sets.

\begin{tabular}{lccccc}
\hline & \multicolumn{2}{c}{ Inter-variance } & & \multicolumn{2}{c}{ Intra-variance } \\
\cline { 2 - 3 } \cline { 6 - 6 } Parameters & $G F D_{\Delta-\Delta \Delta}$ & Sub & & GF $D_{\Delta-\Delta \Delta}$ & Sub \\
\hline Set-1 & 1.75 & 5.68 & & 0.26 & 40.83 \\
Set-2 & 1.53 & 3.68 & & 0.27 & 52.51 \\
\hline
\end{tabular}


excitation information, the $G F D_{\Delta \Delta}$ and $S u b$ features can be used for speaker identification and verification tasks, respectively.

\subsection{Complimentary speaker information from explicit and implicit modelling}

So far we observe that, both implicit and explicit features can be used to model the subsegmental level information. The implicit and explicit features seem to be relatively more effective for identification and verification tasks, respectively. The speaker-specific evidence present in the $G F D_{\Delta-\Delta \Delta}$ and $S u b$ features corresponds to the vocal excitation and hence is different from the vocal tract information. The speaker-specific evidence from $G F D_{\Delta-\Delta \Delta}$ and $S u b$ features can be combined with the state-of-the-art vocal tract features to further improve the recognition accuracy. The improvement in the performance by combining the evidences mostly depends on how much they provide different aspect of speaker-specific evidences to each other. The feature with higher complimentary evidence with the vocal tract features may be good choice for speaker recognition. To verify the complimentary nature of the $G F D_{\Delta-\Delta \Delta}$ and $S u b$ feature with vocal tract information, we employ the score or decision level combination scheme (Hall \& Srihari 1994; Kittler et al 1998). For this, the speaker recognition performance of the vocal tract features is evaluated in the similar experimental condition.

State-of-the-art speaker recognition system mostly use $M F C C$ as the features for vocal tract information. In this work, the $M F C C$ features are extracted using the standard procedure as described in Davis \& Mermelstein (1980), Reynolds \& Rose (1995) and Deller et al (1993). The magnitude spectrum of $20 \mathrm{~ms}$ with a shift of $10 \mathrm{~ms}$ block of speech signal are warped with the Mel-bank filter that consists of 24 filters. Discrete cosine transform is applied to the log-energy filter bank output. First 13 coefficients excluding the zeroth one, and their $\Delta$ and $\Delta \Delta$ values are concatenated to represent the vocal tract features. We call it as $M F C C_{\Delta-\Delta \Delta}$ features. Voice detection activity is performed to eliminate the unvoiced features (Hari Krishnan et al 2006).

The speaker identification and verification results of $M F C C_{\Delta-\Delta \Delta}$ features are given in the first row of the table 5. The results show that, for both identification and the verification tasks the performance of the $M F C C_{\Delta-\Delta \Delta}$ features is better than $G F D_{\Delta-\Delta \Delta}$ and $S u b$ features. This may due to the fact that either $G F D_{\Delta-\Delta \Delta}$ features or $S u b$ does not represent the complete vocal excitation information. The degradation in the speaker identification and verification performances due to noise in case of $M F C C_{\Delta-\Delta \Delta}$ features are $27 \%$ and $226 \%$, respectively, that is more than the $G F D_{\Delta-\Delta \Delta}$ and $S u b$ features. This shows that although, the vocal tract information represented by $M F C C_{\Delta-\Delta \Delta}$ features provides better performance but severely affected by

Table 5. Speaker identification and verification performances of $M F C C_{\Delta-\Delta \Delta}$ and its combination with $G F D_{\Delta-\Delta \Delta}$ and $S u b$ features.

\begin{tabular}{|c|c|c|c|c|c|}
\hline \multirow[b]{3}{*}{ Feature } & & \multicolumn{4}{|c|}{ Performance } \\
\hline & & \multicolumn{2}{|c|}{ Identification } & \multicolumn{2}{|c|}{ Verification } \\
\hline & & Set-1 & Set-2 & Clean & Noisy \\
\hline$M F C C_{\Delta-\Delta \Delta}$ & & 87 & 66 & 6.92 & 22.58 \\
\hline \multirow[t]{2}{*}{$S u b+M F C C_{\Delta-\Delta \Delta}$} & Comb $_{1}$ & 84 & 63 & 6.68 & 29.20 \\
\hline & $\mathrm{Comb}_{2}$ & 89 & 69 & 6.92 & 22.58 \\
\hline \multirow{2}{*}{$G F D_{\Delta-\Delta \Delta}+M F C C_{\Delta-\Delta \Delta}$} & $\operatorname{Comb}_{1}$ & 87 & 68 & 6.27 & 20.96 \\
\hline & $\operatorname{Comb}_{1}$ & 92 & 77 & 6.92 & 18.69 \\
\hline
\end{tabular}


noise. In this situation, the vocal excitation features may help in improving the state-of-the-art recognition performance.

To demonstrate the complimentary nature of $M F C C_{\Delta-\Delta \Delta}$ features and $S u b$ features with the vocal tract features, we use linear and logical OR combination schemes (Hall \& Srihari 1994; Kittler et al 1998). In case of linear combination, the respective scores are weighted by their performances and combined. For example, the LLR of the combined system, $L L R_{S}$, is given by the following relation:

$$
L L R_{s}=\sum_{i=1}^{L} \frac{R_{i}}{\sum_{i=1}^{T} R_{i}} \times L L R_{i},
$$

where, $L$ is the number of systems combined, $L L R_{i}$ and $R_{i}$ are the LLR and identification performance of the $i^{\text {th }}$ system, respectively. In case of verification task, the $R_{i}$ in Eq. (28) is replaced by the reciprocal of the respective EER and then the scores of the combined system is computed accordingly. The simple linear combination scores with predefined weights may also results in a wrong decision (Zheng et al 2007). The potential of the combined system is further verified from the logical $O R$ combination. In this combination scheme, we use the ground truth information for decision. In case of identification, if any one system is giving the correct decision, we consider it as a correct decision. In case of verification, the true scores around the mean provided by the good system are modified based on the information provided by the poor system (Pati \& Prasanna 2011, 2013). The logical OR combination scheme ensures the performance of the good system unaffected and at the same time exploits the evidences provided by the poor system. In this work, linear and logical $O R$ combinations are abbreviated as $C o m b_{1}$ and $\mathrm{COmb}_{2}$, respectively.

The speaker recognition results of $M F C C_{\Delta-\Delta \Delta}$ combined with $S u b$ and $G F D_{\Delta-\Delta \Delta}$ features are given in second and third rows of the table 5, respectively. The DET curves from the verification results using $C o m b_{1}$ and $C o m b_{2}$ schemes are shown in figures 10 and 11, respectively. The results show that in all cases the maximum benefit we can achieve from $G F D_{\Delta \Delta}+M F C C_{\Delta-\Delta \Delta}$ and $S u b+M F C C_{\Delta-\Delta \Delta}$ is better than the individual performance of the $M F C C_{\Delta-\Delta \Delta}$ feature. The improvement in the performance indicates that the decision taken by subsegmental level information is different and complimentary to the vocal tract information. If suitable combination
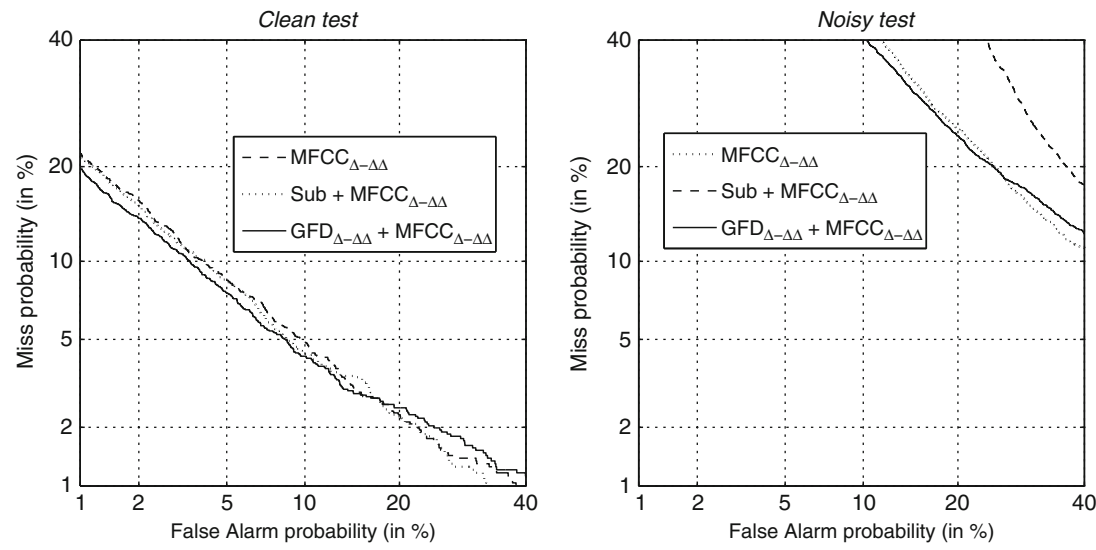

Figure 10. DET curves for $M F C C_{\Delta-\Delta \Delta}$ feature and its combination with $G F D_{\Delta-\Delta \Delta}$ and $S u b$ features using $C o m b_{1}$ scheme. 

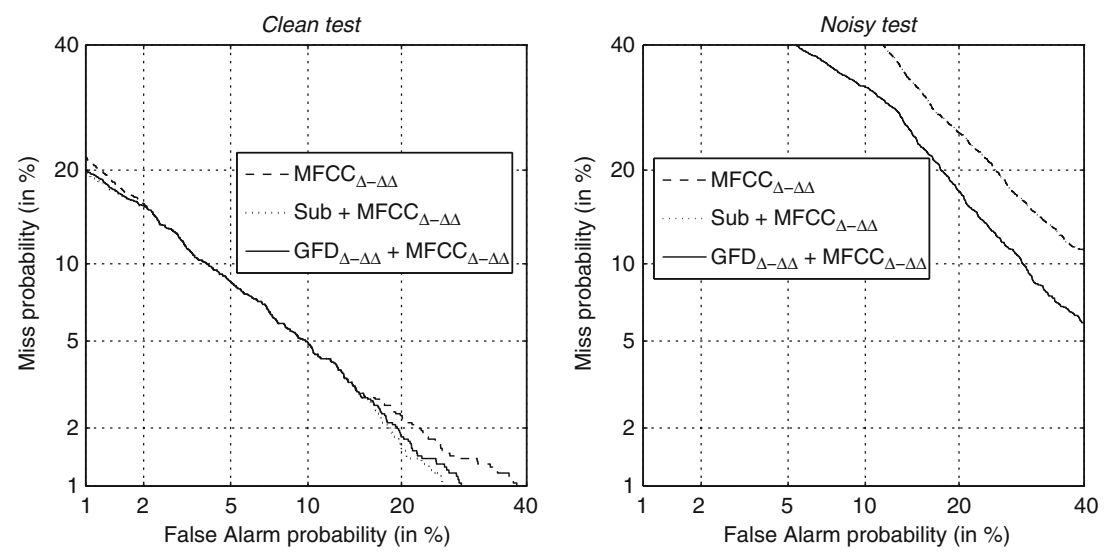

Figure 11. DET curves for $M F C C_{\Delta-\Delta \Delta}$ feature and its combination with $G F D_{\Delta-\Delta \Delta}$ and $S u b$ features using $\mathrm{Comb}_{2}$ scheme.

technique is available then the subsegmental level information modelled by either approaches may help in improving the performance of the $M F C C_{\Delta-\Delta \Delta}$ feature. Further, the complimentary information from the explicit modelling of the subsegmental level information is relatively better than the corresponding implicit approach. For example, the maximum identification accuracy we can achieve in case of $G F D_{\Delta \Delta}+M F C C_{\Delta-\Delta \Delta}$ is $92 \%$ and $77 \%$, as against $89 \%$ and $69 \%$ in case of $S u b+M F C C_{\Delta-\Delta \Delta}$ for Set -1 and Set -2 data sets, respectively. Similarly, in case of the verification task the minimum EER we can achieve in case of $G F D_{\Delta \Delta}+M F C C_{\Delta-\Delta \Delta}$ is $6.27 \%$ and $18.69 \%$, as against $6.68 \%$ and $22.58 \%$ in case of $S u b+M F C C_{\Delta-\Delta \Delta}$ for clean and noisy cases, respectively. This shows that the $G F D_{\Delta-\Delta \Delta}$ provides relatively more complimentary information to the $M F C C_{\Delta-\Delta \Delta}$ feature. Further, the relative degradation in the performance due to noise in case of $G F D_{\Delta \Delta}+M F C C_{\Delta-\Delta \Delta}$ is less than $S u b+M F C C_{\Delta-\Delta \Delta}$ features. For example, for $C o m b_{1}$ scheme, the relative degradation in the identification accuracy from Set -1 to Set -2 of $G F D_{\Delta \Delta}+M F C C_{\Delta-\Delta \Delta}$ is $16 \%$, as against $23 \%$ in case of $S u b+M F C C_{\Delta-\Delta \Delta}$. Similarly, in case of the verification task the relative degradation in the EER from clean to noisy speech in case of $G F D_{\Delta-\Delta \Delta}+G F D_{\Delta-\Delta \Delta}$ feature is $234 \%$, as against $337 \%$ in case of $S u b+M F C C_{\Delta-\Delta \Delta}$ feature. Since the $G F D_{\Delta-\Delta \Delta}$ feature provides relatively more complimentary and robust information to $M F C C_{\Delta-\Delta \Delta}$ feature, it is suggested that explicit modelling of the subsegmental level information by using the LF parameters together with their dynamics may be a good choice for speaker recognition task.

To summarize, the subsegmental excitation information can be modelled by explicit and implicit approaches. In case of implicit modelling, processing the LP residual blocks directly is effective for speaker identification task and computationally more intensive. For the explicit modelling, the use of the LF parameters computed using the proposed approach is effective for speaker verification task but computationally less intensive. The speaker-specific evidence from both the modelling approaches is different and complimentary to the vocal tract information. For both speaker identification and verification tasks, the speaker-specific evidence from the explicit modelling approach is relatively more complimentary to vocal tract feature to further improve the recognition performance. We concluded that explicit modelling of the subsegmental level information using the LF parameters computed using the proposed approach may be a better choice for speaker recognition task. 


\section{Summary and conclusion}

In this work, the speaker-specific subsegmental excitation information is modelled explicitly by characterizing the GFD using LF parameters and a comparison is made with corresponding implicit modelling. A method proposed significantly reduces the computational complexity for measurement of the LF parameters. The statistical distribution of these parameters value from different speakers demonstrates their speaker-specific nature. The feature representation of the LF parameters together with their dynamics provide useful information for speaker recognition. Implicit modelling of the subsegmental level information is performed by processing the LP residual in blocks of $5 \mathrm{~ms}$ with a shift of $2.5 \mathrm{~ms}$ duration. May be due to small intra-speaker variation, explicit approach is found to be useful for verification task. On the other hand, due to large inter-speaker variation, implicit approach is found to be useful for identification task. Individually both implicit and explicit approaches provide recognition accuracy that is relatively poor but provide complimentary speaker-specific evidence to the conventional vocal tract information to further improve the performance. The speaker-specific evidence from the explicit modelling approach provides relatively more complimentary and robust information to vocal tract features. These observations suggest that, explicit modelling of the subsegmental level information by the proposed approach may be more effective for speaker recognition task.

Even if, there are noticeable differences in the LF parameters across speakers, this would not mean that they represent the actual glottal characteristic of the speaker. Because, the LF model of the glottal flow is a much reduced version of the actual glottal flow (Plumpe et al 1999). More accurate modelling of the glottal flow may help in the better representation of the subsegmental level information explicitly. Further, both implicit and explicit modelling of the subsegmental level information do not contribute much to vocal tract features. This should not be confused with the worth of combined use of subsegmental excitation information with vocal tract features. Because, the performance of the combined system also depends on the combination scheme employed. New combination technique needs to be developed to exploit the same. For this, amount of representative and discriminating information captured by each feature measurements may be useful (Padmanabhan \& Murthy 2010).

\section{References}

Ananthapadmanabha T V and Fant G 1982 Calculation of true glottal flow and its components. Speech Commun. 1: 167-184

Ananthapadmanabha T V and Yegnanarayana B 1979 Epoch extraction from linear prediction residual for identification of closed glottis interval. IEEE Trans. on Acoust., Speech and Signal Process. ASSP-27: 309-319

Atal B S 1972 Automatic speaker recognition based on pitch contours. J. Acoust. Soc. Amer. 52(6): 16871697

Atal B S 1976 Automatic recognition of speakers from their voices. Proc. IEEE 64(4): 460-475

Bimbot F, Bonastre J-F, Fredouille C et al 2004 A tutorial on text-independent speaker verification. EURASIP Journal on Applied Signal Processing 4: 430-451

Campbell J P Jr 1997 Speaker recognition: A tutorial. Proc. IEEE 85(9): 1437-1462

Carlson R, Fant G, Gobl C, Granstrom B, Karlsson I and Lin Q-G 1989 Voice source rules for text-to-speech synthesis. In: Int. conf. on Acoust. Speech and Signal Process. (ICASSP) vol. 1, Glasgow, Scotland, $223-226$

Cohen L 1995 Time-frequency analysis: theory and application, ser. Signal Processing Series. Englewood Cliffs: Prentice Hall 
Davis S B and Mermelstein P (1980) Comparison of parametric representations for monosyllabic word recognition in continuously spoken sentences. IEEE Trans. Acoust. Speech and Signal Process. 28(28): 357-366

Deller J R Jr, Hansen J H L and Proakis J G 1993 Discrete-time processing of speech signal, 2nd ed. New York: IEEE Press

Duda R O and Hart P E 2001 Pattern Classification, 2nd ed. Willy

Ezzaidi H and Rouat J 2004 Pitch and MFCC dependent GMM models for speaker identification systems. In: IEEE int. conf. on Electrical and Computer Engg., vol. 1

Furui S 1981a Cepstral analysis technique for automatic speaker verification. IEEE Trans. Acoust. Speech, and Signal Process. 29(2): 254-272

Furui S 1981b Comparison of speaker recognition methods using static features and dynamic features. IEEE Trans. Acoust., Speech and Signal Process. ASSP-29(3): 342-350

Furui S 2005 Fifty years progress in speech and speaker recognition research. Proc. SPECOM. Patras, Greece, $1-9$

Haeb-Umbach R 1999 Investigation on inter-speaker variability in the feature space. In: Int. conf. on Acoust. Speech and Signal Process. (ICASSP), Phoenix, AZ, 397-400

Hall J J and Srihari S N 1994 Decision combination in multiple classifier systems. IEEE Trans. Patt. Anal. and Mach. Intell. 16: 66-75

Hari Krishnan P, Padmanabhan R and Murthy H A 2006 Robust voice activity detection using group delay functions. In: Proc. IEEE International Conference on Industrial Technology, 2603-2607

Hayakawa S, Takeda K and Itakura F 1997 Speaker identification using harmonic structure of lpresidual spectrum. Biometric personal Aunthentification, Lecture notes, vol. 1206. Springer, Berlin, 253260

Huang W, Chao J and Zhang Y 2008 Combination of pitch and MFCC GMM supervectors for speaker verification. In: IEEE int. conf. on Audio, Language and Image Process. (ICALIP), 1335-1339

Iseli M R and Alwan A 2000 Inter- and intra-speaker variability of glottal flow derivative. In: Int. conf. on Spoken Language Processing (ICSLP, 2000), Beijing, Chaina

Karlsson I 1988 Glottal waveform parameters for different speaker types. STL-QPSR, 29(2-3): 61-67

Kittler J, Hatef M, Duin R P W and Matas J 1998 On combining classifiers. IEEE Trans. Patteren Analysis and Machine Intelligence 20(3): 226-239

Kominek J and Black A 2004 CMU-Arctic speech database. In: 5th ISCA Speech Synthesis Workshop. Pittsburg, PA, 223-224

Makhoul J 1975 Linear prediction: A tutorial review. Proc. IEEE 63(4): 561-580

Martin A, Doddington G, Kamm T, Ordowski M and Przybocki M 1997 The DET curve in assessment of detection task performance. In: Proc. Eur. Conf. on Speech Communication Technology, vol. 4. Rhodes, Greece, 1895-1898

Mary L and Yegnanarayana B (2008) Extraction and representation of prosodic features for language and speaker recognition. Speech Commun. 50: 782-796

Murty K S R and Yegnanarayana B 2006 Combining evidence from residual phase and MFCC features for speaker recognition. IEEE Signal Process. Lett. 13(1): 52-55

Murthy K S R and Yegnanarayana B 2008 Epoch extraction from speech signal. IEEE Trans. Audio Speech and Language Process. 16(8): 1602-1613

Murthy K S R and Yegnanarayana B 2009 Characterization of glottal activity from speech signal. IEEE Signal Process. Lett. 16(6): 469-472

Murty K S R, Prasanna S R M and Yegnanarayana B 2004 Speaker specific information from residual phase. In: Int. Conf. on Signal Proces. and Comm. (SPCOM)

Naylor P A, Kounoudes A, Gudnason J and Brookes M 2007 Estimation of glottal closure instants in voiced speech using the dypsa algorithm. IEEE Trans. Audio Speech and Language Process. 15(1):34-43

Padmanabhan R and Murthy H A 2010 Acoustic feature diversity and speaker verification. In: INTERSPEECH 2010, Makuhari, Chiba, Japan, 2010-2013

Pati D and Prasanna S R M 2008 Non-parametric vector quantization of excitation source information for speaker recognition. In: Proc. IEEE TENCON, 1-4 
Pati D and Prasanna S R M 2010 Speaker information from subband energies of linear prediction residual. In: Proc. NCC 2010, 1-4

Pati D and Prasanna S R M 2011 Subsegmental, segmental and suprasegmental processing of linear prediction residual for speaker information. Int. J. of Speech Technology, Springer 14(1): 49-63

Pati D and Prasanna S R M 2013 Processing of linear prediction residual in spectral and cepstral domains for speaker information. Proceedings in Communicated to SADHNA Academy Proceedings in Engineering Sciences, Springer

Plumpe M D, Quatieri T F and Reynolds D A 1999 Modelling of glottal flow derivative waveform with application to speaker identification. IEEE Trans. Speech and Audio Process. 7(5): 569-586

Prasanna S R M, Gupta C S and Yegnanarayana B (2006) Extraction of speaker-specific excitation information from linear prediction residual of speech. Speech Commun. 48: 1243-1261

Pruzansky S and Mathews M V 1964 Talker-recognition procedure based on analysis of variance. J. Acoust. Soc. Amer. 36(11): 2041-2047

Przybocky M and Martin A 2000 The NIST-1999 speaker recognition evaluation- An overview. Digital Signal Processing 10: 1-18

Przybocky M and Martin A 2003 Nist speaker recognition evaluation plan. In: Proc. NIST Speaker Recognition Workshop, College Park, MD

Qi Y and Bi N 1994 A simplified approximation of the four-parameter LF model of voice source. J. Acoustic. Soc. Amer. 96(2): 1182-1185

Reynolds D A 1994 Experimental evaluation of features for robust speaker identification. IEEE Trans. Speech Audio Process. 2(4): 639-643

Reynolds D A and Rose R C 1995 Robust text-independent speaker identification using gaussian mixture speaker models. IEEE Trans. Speech and Audio Process. 3(1): 4-17

Strik H 1998 Automatic parameterization of differentiated glottal flow: Comparing methods by means of synthetic flow pulses. J. Acoust. Soc. Amer. 103(5): 2659-2669

Thevenaz P and Hugli H 1995 Usefulness of the LPC-residue in text-independent speaker verification. Speech Commun. 17: 145-157

Veldhuish R 1998 A computationally efficient alternative for the Liljencrants-Fant model and its perceptual evaluation. J. Acoust. Soc. Amer. 103(1): 566-571

Wang N, Ching P C and Lee T 2009 Exploration of vocal excitation modulation features for speaker recognition. In: Proc. INTERSPEECH-09, Brighton UK, 892-895

Wolf J J 1972 Efficient acoustic parameters for speaker recognition. J. Acoust. Soc. Amer. 51(2): 20442055

Yegnanarayana B and Veldhuis R N J 1998 Extraction of vocal-tract system characterstics from speech signals. IEEE Trans. Speech Audio Proc. 6(4): 313-327

Yegnenarayana B and Murthy K S R 2009 Event based instantaneous fundamental frequency estimation from speech signals. IEEE Trans. Audio, Speech and Language Process. 17(4): 614-624

Yegnanarayana B and Prasanna S R M 2010 Analysis of instantaneous F0 contours from two speakers mixed signal using zero frequency filtering. In: Int. Conf. on Acoust. Speech and Signal Process. (ICASSP), Dallas, Texas, USA, 5074-5077

Yegnanarayana B, Prasanna S R M, Zachariah J M, Gupta C S 2005 Combining evidences from source, suprasegmental and spectral features for fixed-text speaker verification study. IEEE Trans. on Speech and Audio Process. 13(4): 575-582

Yegnanarayana B, Reddy K S and Kishore S P 2001 Source and systsem feature for speaker recognition using AANN Models. Proc. IEEE Int. Conf. Acoust. Speech and Signal Process. Salt Lake City, UT, USA, 409-412

Zheng N, Lee T and Ching P C 2007 Integration of complimentary acoustic features for speaker recognition. IEEE Signal Process. Lett. 14(3): 181-184 